\title{
1 Large-Scale Uniform Analysis of Cancer Whole Genomes in Multiple
} 2 Computing Environments

4 Christina K. Yung ${ }^{1, *}$, Brian D. O'Connor ${ }^{1,2, *}$, Sergei Yakneen ${ }^{1,3, *}$, Junjun Zhang ${ }^{1, *}$, Kyle Ellrott ${ }^{4}$, 5 Kortine Kleinheinz ${ }^{5,6}$, Naoki Miyoshi ${ }^{7}$, Keiran M. Raine ${ }^{8}$, Romina Royo $^{9}$, Gordon B. Saksena ${ }^{10}$, 6 Matthias Schlesner ${ }^{5}$, Solomon I. Shorser ${ }^{1}$, Miguel Vazquez ${ }^{11}$, Joachim Weischenfeldt ${ }^{3,12}$, Denis 7 Yuen ${ }^{1}$, Adam P. Butler ${ }^{8}$, Brandi N. Davis-Dusenbery ${ }^{13}$, Roland Eils ${ }^{14,6}$, Vincent Ferretti ${ }^{1}$, Robert L. 8 Grossman $^{15}$, Olivier Harismendy ${ }^{16,17}$, Youngwook Kim ${ }^{18}$, Hidewaki Nakagawa ${ }^{19}$, Steven J. 9 Newhouse $^{20}$, David Torrents ${ }^{9,21}$,Lincoln D. Stein ${ }^{1,22, \$}$ on behalf of the PCAWG Technical Working 10 Group $^{23}$ and the PCAWG Network

* These authors contributed equally to this work.

$\neq$ Corresponding author: lincoln.stein@gmail.com

${ }^{1}$ Informatics and Biocomputing Program, Ontario Institute for Cancer Research, Toronto, Ontario, M5G 0A3, Canada. ${ }^{2}$ UC Santa Cruz Genomics Institute, University of California Santa Cruz, Santa Cruz, California, 95065, USA. ${ }^{3}$ Genome Biology Unit, European Molecular Biology Laboratory, Heidelberg, Baden-Württemberg, 69120, Germany. ${ }^{4}$ Department of Computational Biology, Oregon Health and Science University, Portland, Oregon, 97239, USA. ${ }^{5}$ Division of Theoretical Bioinformatics, German Cancer Research Center (DKFZ), Heidelberg, Baden-Württemberg, 69120, Germany. ${ }^{6}$ Department for Bioinformatics and Functional Genomics, Institute for Pharmacy and Molecular Biotechnology and BioQuant, Heidelberg University, Heidelberg, Baden-Württemberg, 69120, Germany. ${ }^{7}$ Human Genome Center, Institute of Medical Science, University of Tokyo, Tokyo, 108-8639, Japan. ${ }^{8}$ Cancer Ageing and Somatic Mutation Programme, Wellcome Trust Sanger Institute, Hinxton, Cambridgeshire, CB10 1SA, United Kingdom. ${ }^{9}$ Department of Life Sciences, Barcelona Supercomputing Center, Barcelona, Catalunya, 8034, Spain. ${ }^{10}$ Cancer Program, Broad Institute of MIT and Harvard, Cambridge, Massachusetts, 02142, USA. ${ }^{11}$ Structural Computational Biology Group, Centro Nacional de Investigaciones Oncologicas, Madrid, Madrid, 28029, Spain. ${ }^{12}$ BRIC/Finsen Laboratory, Rigshospitalet, Copenhagen, 2200, Denmark. ${ }^{13}$ Seven Bridges, Cambridge, Massachusetts, 02142, USA. ${ }^{14}$ Theoretical Bioinformatics, German Cancer Research Center (DKFZ), Heidelberg, Baden-Württemberg, 69120, Germany. ${ }^{15}$ Center for Data Intensive Science, University of Chicago, Chicago, Illinois, 60637, USA. ${ }^{16}$ Department of Medicine, University of California San Diego, San Diego, California, 92093, USA. ${ }^{17}$ Moores Cancer Center, Department of Medicine, Division of Biomedical Informatics, University of California San Diego, San Diego, California, 92093, USA. ${ }^{18}$ Samsung Advanced Institute of Health Science and Technology, Sungkyunkwan University, School of Medicine, Seoul, 135-710, South Korea. ${ }^{19}$ Laboratory for Genome Sequencing Analysis, RIKEN Center for Integrative Medical Sciences, Tokyo, 108-8639, Japan. ${ }^{20}$ Technical Services Cluster, European Molecular Biology Laboratory, European Bioinforamtics Institute, Hinxton, Cambridge, CB10 1SD, United Kingdom. ${ }^{21}$ Institució Catalana de Recerca i Estudis Avançats, Barcelona, Catalunya, 8010, Spain. ${ }^{22}$ Department of Molecular Genetics, University of Toronto, Toronto, Ontario, M5S 1A1, Canada. ${ }^{23}$ Full lists of members and affiliations appear at the end of the paper. 
$\underline{\text { Abstract }}$

41 The International Cancer Genome Consortium (ICGC)'s Pan-Cancer Analysis of Whole Genomes

42 (PCAWG) project aimed to categorize somatic and germline variations in both coding and non-

43 coding regions in over 2,800 cancer patients. To provide this dataset to the research working

44 groups for downstream analysis, the PCAWG Technical Working Group marshalled $~ 800 \mathrm{~TB}$ of

45 sequencing data from distributed geographical locations; developed portable software for uniform

46 alignment, variant calling, artifact filtering and variant merging; performed the analysis in a

47 geographically and technologically disparate collection of compute environments; and

48 disseminated high-quality validated consensus variants to the working groups. The PCAWG

49 dataset has been mirrored to multiple repositories and can be located using the ICGC Data Portal.

50 The PCAWG workflows are also available as Docker images through Dockstore enabling

51 researchers to replicate our analysis on their own data.

\section{Introduction}

53 The International Cancer Genome Consortium (ICGC)/The Cancer Genome Atlas (TCGA) Pan-

54 Cancer Analysis of Whole Genomes (PCAWG) study has characterized the pattern of mutations

55 in over 2,800 cancer whole genomes. Extending TCGA Pan-Cancer analysis project, which

56 focused on molecular aberrations in protein coding regions only ${ }^{1}$, PCAWG undertook the study of

57 whole genomes, allowing for the discovery of driver mutations in cis-regulatory sites and non-

58 coding RNAs, examination of the patterns of large-scale structural rearrangements, identification

59 of signatures of exposure, and elucidation of interactions between somatic mutations and germline

60 polymorphisms.

61 The PCAWG dataset comprises a total of 5,789 whole genomes of tumors and matched normal

62 tissue spanning 39 tumor types. The tumor/normal pairs came from a total of 2,834 donors 
63 collected and sequenced by 48 sequencing projects across 14 jurisdictions (Supplementary Fig. 1).

64 In addition, RNA-Seq profiles were obtained from a subset of 1,284 of the donors ${ }^{2}$. While the

65 individual sequencing projects contributing to PCAWG had previously identified genomic variants

66 within their individual cancer cohorts, each project had used their own preferred methods for read

67 alignment, variant calling and artifact filtering. During initial evaluation of the data set, we found

68 that the different analysis pipelines contributed high levels of technical variation, hindering

69 comparisons across multiple cancer types ${ }^{3}$. To eliminate the variations arising from non-uniform

70 analysis, we reanalyzed all samples starting with the raw sequencing reads and using a

71 standardized set of alignment, variant calling and filtering methods. These "core" workflows

72 yielded uniformly analyzed genomic variants for downstream analyses by various PCAWG

73 working groups. A subset of these variants were validated through targeted deep sequencing to

74 estimate the accuracy of our approach ${ }^{4}$.

75 To create this uniform analysis set, multiple logistic and technical challenges had to be overcome.

76 First, projects participating in the PCAWG study employed their own metadata conventions for

77 describing their raw sequencing data sets. Hence, we had to establish a PCAWG metadata standard

78 suitable for all the participating projects. Second, and more significantly, the data was large in size

79 -- 800TB of raw sequencing reads -- and distributed geographically across the world. During

80 realignment, the data transiently doubled in size, and after final variant calling and other

81 downstream analysis, the full data set reached nearly 1PB. Furthermore, the compute necessary to

82 fully harmonize the data was estimated at more than 30 million core-hours. Both the storage and

83 compute requirements made it impractical to complete the analysis at any single research institute.

84 In addition, legal constraints across the various jurisdictions imposed restrictions as to where

85 personal data could be stored, analyzed and redistributed ${ }^{5}$. Hence, we needed a protocol to spread 
86 the compute and storage resources across multiple commercial and academic compute centers.

87 This requirement, in turn, necessitated the development of analysis pipelines that would be

88 portable to different compute environments and yield consistent analysis results independent of

89 platform. With multiple analysis pipelines running simultaneously in multiple compute

90 environments, the assignment of workload, tracking of progress, quality checking of data and

91 dissemination of results all required sophisticated and flexible planning.

92 Our approach to tackling these challenges was unique and substantially different from previous

93 large-scale genome analysis endeavors. First, as a collaborative effort among a wide range of

94 institutions not backed by a centralized funding source, a high degree of coordination among a

95 large task force of volunteer software engineers, bioinformaticians and computer scientists was

96 required. Second, the project fully embraced the use of both public and private cloud compute

97 technologies while leveraging established high-performance computing (HPC) infrastructures to

98 fully utilize the compute resources contributed by the partner organizations. The cloud technology

99 platforms we utilized included both Infrastructure as a Service (IaaS): OpenStack, Amazon Web

100 Services and Microsoft Azure; and Platform as a Service (PaaS): Seven Bridges (SB). Lastly, the

101 project made heavy use of Docker, a new lightweight virtualization technology that ensured

102 workflows, tools and infrastructure would work identically across the large number of compute

103 environments utilized by the project.

104 Utilizing the compute capacity contributed by academic HPC, academic clouds and commercial

105 clouds (Table 1), we were able to complete a uniform analysis of the entire set of 5,789 whole

106 genomes in just over 23 months (Figure 1). Figure 3 illustrates the three broad phases of the project:

107 (1) Marshalling and upload of the data into data analysis centres (3 months); (2) Alignment and

108 variant calling (18 months); and (3) Quality filtering, merging, synchronization and distribution of 
109 the variant calls to downstream research groups (2 months). A fourth phase of the project, in which

110 PCAWG working groups used the uniform variant calls for downstream analysis, such as cancer

111 driver discovery, began in the summer of 2016 and continued through the first two quarters of 1122017.

113 The following sections will describe the technical solutions used to accomplish each of the phases

114 of the project.

\section{Phase 1: Data Marshalling and Upload}

116 A significant challenge for the project was that at its inception, a large portion of the raw read 117 sequencing data had yet to be submitted to a read archive and thus had no standard retrieval 118 mechanism. In addition, the metadata standards for describing the raw data varied considerably

119 from project to project. For this reason, we asked the participating projects to prepare and upload 120 the $774 \mathrm{~TB}$ of raw whole genome sequencing (WGS) data and $27 \mathrm{~TB}$ raw RNA-seq data into a 121 series of geographically distributed data repositories, each running a uniform system for registering 122 the data set, accepting and validating the raw read data and standardized metadata.

123 We utilized seven geographically distributed data repositories located at: (1) Barcelona

124 Supercomputing Centre (BSC), (2) European Bioinformatics Institute (EMBL-EBI) in the UK, (3)

125 German Cancer Research Center (DKFZ) in Germany; (4) the University of Tokyo in Japan; (5)

126 Electronics and Telecommunications Research Institute (ETRI) in South Korea; (6) the Cancer

127 Genome Hub (CGHub) and (7) the Bionimbus Protected Data Cloud (PDC) in the USA (Figure 2 128 and Suppl Table 1).

129 To accept and validate sequence set uploads, each data repository ran a commercial software 130 system, GNOS (Annai Systems). We chose GNOS because of the heavy testing it had previously 
131 received as the engine powering TCGA CGHub, and its support for validation of metadata

132 according to the Sequence Read Archive (SRA) standard and file submission, strong user

133 authentication and encryption, as well as its highly optimized data transfer protocol ${ }^{6}$. Each of the

134 seven data centers initially allocated several hundred terabytes of storage to accept raw sequencing

135 data from submitters within the region. The data centers also provided co-located compute

136 resources to perform alignment and variant calling on the uploaded data.

137 Genomic data uploaded to the GNOS repositories was accompanied with detailed and accurate 138 metadata to describe the cancer type, sample type, sequencing type and other attributes for 139 managing and searching the files. We required that identifiers for project, donor, sample follow a

140 standardized convention such that validation and auditing tools could be implemented. Most of the

141 naming conventions in PCAWG were adopted from the well established ICGC data dictionary

142 (http://docs.icgc.org/dictionary/about/).

143 Since most member projects at the time of upload already had sequencing reads aligned and

144 annotated using their own metadata standards, a non-trivial effort was required to prepare the

145 sequencing data for submission to GNOS. Each member project had to (1) prepare lane-level

146 unaligned reads in BAM format, (2) reheader the BAM files with metadata following the PCAWG

147 conventions, (3) generate metadata XML files, and (4) upload the BAM files along with the

148 metadata XML files to GNOS. To facilitate this process, we developed the PCAP-core tool

149 (https://github.com/ICGC-TCGA-PanCancer/PCAP-core) to extract the metadata from the BAM

150 headers, validate the metadata, transform the metadata into the XML files conforming to the SRA

151 specifications, and submitting the BAM files along with the metadata XML files to GNOS. 


\section{Phase 2: Sequence Alignment and Variant Calling}

154 We began the process of sequence alignment about two months after the uploading process had

155 begun. Both tumor and matched normal reads were subjected to uniform sequence alignment using

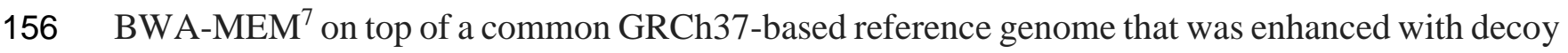

157 sequences, viral sequences, and the revised Cambridge reference genome for the mitochondria.

158 Efforts by the project QC group demonstrated that employing multiple variant callers in ensemble

159 fashion improved calling sensitivity ${ }^{3}$, thus the aligned tumor/normal pairs were subjected to

160 somatic variant calling using three "best practice" software pipelines. These pipelines were

161 developed by the Sanger Institute ${ }^{8-11}$; jointly by $\mathrm{DKFZ}^{12}$ and the European Molecular Biology

162 Laboratory $(\mathrm{EMBL})^{13}$; and the Broad Institute ${ }^{14}$ with contribution from MD Anderson Cancer

163 Center-Baylor College of Medicine ${ }^{15}$. Each pipeline represents the best practices from the

164 authoring organizations and include the current versions of each institute's flagship tools. Each

165 pipeline consists of multiple software tools for calling of single and multiple nucleotide variants

166 (SNVs and MNVs), small insertions/deletions (indels), structural variants (SVs) and somatic copy

167 number alterations (SCNAs). The minimum compute requirements, median runtime and the

168 analytical algorithms for each pipeline are shown in Table 2.

169 When possible, both the alignment and variant calling pipelines were executed in the same regional

170 compute centers to which the data sets were uploaded. As the project progressed, we utilized

171 additional compute resources from AWS, Azure, iDASH, the Ontario Institute for Cancer

172 Research (OICR), the Sanger Institute, and Seven Bridges (Figure 2). These centers computed on

173 data sets located in the same region to optimize data transfer. Over the course of the project, some

174 centers outpaced others and we rebalanced data sets as needed to use resources as efficiently as 
175 possible. Figure 1 shows the progress of the analytic pipelines with more details shown in

176 Supplementary Figures 2-6.

\section{Phase 3: Variant merging, filtering, and synchronization}

178 Following the completion of the three variant calling workflows, variants were passed to an

179 additional pipeline referred as the "OxoG workflow". This pipeline filtered out oxidative artifacts

180 in SNVs using the OxoG algorithm ${ }^{16}$, normalized indels using the bcftools "norm" function,

181 annotated genomic features for downstream merging of variants, and generated one "minibam"

182 per specimen using the VariantBam algorithm ${ }^{17}$. Minibams are a novel format for representing the

183 evidence that underlies genomic variant calls. Read pairs spanning a variant within a specified

184 window were extracted from the whole genome BAM to generate the minibam. The windows we

185 chose were +/- 10 base pairs (bp) for SNVs, +/- 200 bp for indels, and +/- 500 bp for SV

186 breakpoints. The resulting minibams are about $0.5 \%$ of the size of whole genome BAMs, totalling

187 to about four terabytes for all PCAWG specimens, making it much easier to download and store

188 for the purpose of inspecting variants and their underlying read evidence.

189 Following filtering, we applied a series of merge algorithms to merge variants from the multiple

190 variant calling pipelines into consensus call sets with higher accuracies than the individual

191 pipelines alone. The SNV and indel merge algorithms were developed on the basis of experimental

192 validation of the individual variant calling pipelines using deep targeted sequencing, a process

193 detailed in the PCAWG-1 marker paper ${ }^{4}$. The algorithm for consensus SVs is described in the

194 PCAWG-6 marker paper ${ }^{18}$. The consensus SCNAs were built upon the base-pair breakpoint

195 results from the consensus SVs using a multi-tiered bespoke approach combining results from 6

196 SCNA algorithms ${ }^{19}$. 
197 Following merging, the SNV, indel, SV and SCNA consensus call sets were subjected to intensive

198 examination by multiple groups in order to identify anomalies and artefacts, including uneven

199 coverage of the genome, strand and orientation bias, contamination with reads from non-human

200 species, contamination of the library with DNA from an unrelated donor, and high rates of common

201 germline polymorphisms among the somatic variant calls ${ }^{4,11}$. In keeping with our mission to

202 provide a high-quality and uniformly annotated data set, we developed a series of filters to annotate

203 and/or remove these artefacts. Tumor variant call sets that were deemed too problematic to use for

204 downstream analysis were placed on an "exclusion list" (353 specimens, 176 donors). In addition,

205 we established a "grey list" (150 specimens, 75 donors), of call sets that had failed some tests but

206 not others and could be used, with caution, for certain types of downstream analysis. The criteria

207 for classifying callsets into exclusion and grey list are described in more detail in the PCAWG-1

208 paper $^{10}$.

209 Following the filtering steps, we used GNOS to synchronize the aligned reads and variant call sets

210 among a small number of download sites for use by PCAWG downstream analysis working groups

211 (Suppl Table 2). We also provided login credentials to members of PCAWG working groups for

212 compute cloud-based access to the aligned read data across several of the regional data analysis

213 centers, which avoided the overhead of downloading the data.

\section{Software and Protocols}

215 This section describes the software and protocols developed for this project in more detail. All the

216 software that we created for this project is available for use by any research group to conduct

217 similar cloud-based cancer genome analyses economically and at scale. 
220 The metadata describing the donors, specimens, raw sequencing reads, WGS and RNA-Seq

221 alignments, variant calls from the three pipelines, OxoG-filtered variants, and mini-BAMs were

222 collected from globally distributed GNOS repositories, consolidated and indexed nightly using

223 ElasticSearch (https://www.elastic.co) in a specially designed object graph model. This centrally

224 managed metadata index was a key component of our operations and data provenance tracking.

225 First, the metadata index was critical for tracking the status of each sequencing read set and for

226 scheduling the next analytic step. The index also tracked the current location of each BAM and

227 variant call set, allowing the pipelines to access the needed input data efficiently. Second, the

228 metadata index provided the basis for a dashboard (http://pancancer.info) for all stakeholders to

229 track day-to-day progress of each pipeline at each compute site. By reviewing the throughput of

230 each compute site on a daily basis, we were able to identify issues early and to assign work

231 accordingly to keep our compute resources productive. Third, the metadata index was also used

232 by the ICGC Data Coordination Centre (DCC) to transfer PCAWG core datasets to long-term

233 genomic data archive systems. Finally, the metadata index was imported into the ICGC Data Portal

234 (https://dcc.icgc.org) to create a faceted search for PCAWG data allowing users to quickly locate

235 data based on queries about the donor, cancer type, data type or data repositories.

\section{Docker Containers \& Consonance}

237 Given that the compute resources donated to the PCAWG project were a mix of cloud and HPC

238 environments, we required a mechanism to encapsulate the analytical workflows to allow them to

239 run smoothly across a wide variety of compute sites. The approaches we used evolved over time

240 to incorporate better ways of abstracting and packaging tools to facilitate this portability. Initially,

241 we used SeqWare workflow execution engine ${ }^{20}$ for bundling software and executing workflows, 
242 but this system required extensive and time consuming setup for the worker virtual machines

243 (VMs). Later, we adopted Docker (http://www.docker.com) as a key enabling technology for

244 running workflows in an infrastructure-independent manner. As a lightweight, infrastructure-

245 agnostic containerization technology, Docker allowed PCAWG pipeline authors to fully

246 encapsulate tools and system dependencies into a portable image. This included the fleet of VMs

247 on commercial and academic clouds, as well as the project's HPC clusters that were modified to

248 support Docker containers. Each of our major pipelines was encapsulated in a single Docker

249 image, along with a suitable workflow execution engine, reference data sets, and software libraries

250 (Table 2).

251 Another key component of the PCAWG software infrastructure stack was cloud-agnostic

252 technology to provision virtual machines on both academic and commercial clouds. Our initial

253 attempts to scale the analytic pipelines across multiple cloud systems were complicated by

254 transient failures in many of the academic cloud environments, subtle differences between

255 seemingly identical clouds, and misconfigured services within the clouds. Initially, we attempted

256 to replicate within the clouds standard components of conventional HPC environments, including

257 shared file systems and cluster load balancing systems. However, we quickly learned that these

258 perform poorly in the dynamic environments of the cloud. After several design iterations, we

259 developed Consonance (https://github.com/consonance), a cloud-agnostic provisioning and

260 queueing platform. For each of the cloud platforms in use in PCAWG, including OpenStack,

261 VMWare, AWS, and Azure, Consonance provided a queue where work scheduling was decoupled

262 from the worker nodes. As the fleet of working nodes shrank or expanded, each queue queried the

263 centralized metadata index to obtain the next batch of tasks to execute. Consonance then created

264 and maintained a fleet of worker VMs, launched new pipeline jobs, detected and relaunched failed 
265 VMs, and reran workflows as needed. Consonance allowed us to dynamically allocate cloud

266 resources depending on the workload at hand, and even interacted with the AWS spot marketplace

267 to minimize our commercial cloud costs.

268 The Operations: whitelist, work queue, cloud shepherds

269 For the duration of the project, several personnel were required to operate the Docker images,

270 Consonance and the metadata index effectively (Figure 4). Each compute environment was

271 managed by a "cloud shepherd" responsible for completing the workflows on a set of pre-assigned

272 donors or specimens. All the HPC environments (BSC, DKFZ, UTokyo, UCSC, Sanger) were

273 shepherded by personnel local to the institute who were already familiar with the specific file

274 systems and work schedulers, and obtained technical support from their local system

275 administrators. The majority of the cloud environments (AWS, Azure, DKFZ, EMBL-EBI, ETRI,

276 OICR, PDC) granted tenancy to OICR whose personnel acted as cloud shepherds. The other clouds

277 (iDASH, SB), newly launched at the time, assigned their own cloud shepherds who also tested and

278 fine tuned their environments in the process.

279 A project manager acted as the point of contact for all the cloud shepherds to report any technical

280 issues and progress, such that the overall availability of compute resources and throughput at any

281 time point could be estimated. Combining this knowledge with the information from the

282 centralized metadata index, the project manager assigned donors and workflows to compute

283 environments in the form of "whitelists" on a weekly basis. Cloud shepherds then added the

284 whitelist of donors to their workflow queue for execution. This approach allowed us to be agile in 285 responding to data availability disruptions, planned or unplanned downtime while optimizing data

286 transfer and operations throughput. 
287 While quotas shifted throughout the duration of the analysis, as demands and workloads on the

288 individual centers changed, the overall peak commitment received was on the order of the 15,000

289 cores, approximately 60TB of RAM, and a peak usage of $\sim 630$ virtual machines.

290 Software Distribution through Dockstore

291 The workflows used during PCAWG production include several PCAWG-specific elements that 292 may limit their usability by researchers outside of the project. To facilitate the long term usage of 293 these workflows by a broad range of cancer genomic researchers, we have simplified the tools to 294 make most workflows standalone (Suppl Table 4). These Docker-packaged workflows have been 295 extensively tested for their reproducibility and are registered on the Dockstore ${ }^{21}$ 296 (http://dockstore.org), a service compliant with Global Alliance for Genomics and Health 297 (GA4GH) standards to provide computational tools and workflows through Docker and described 298 with Common Workflow Language 22 (CWL). This enables other researchers to run the workflows 299 on their own data, extend their utility, and replicate the work we have done in any CWL-compliant 300 environment. By running the identical PCAWG workflows on their own data, researchers will be 301 able to make direct comparisons and add to the existing PCAWG dataset.

302 The Docker-packaged BAM alignment and variant calling workflows were tested in different 303 cloud environments and found to be easy to enact by third parties. Some discrepancies with the 304 official data were observed and attributed to improvements in the underlying software (Sanger, 305 Delly) or to the stochastic nature of the software, and deemed to have a low overall impact. Despite 306 not achieving a completely identical results, the reproducibility of the process is satisfactory, 307 especially considering that it involves software developed independently by different teams. 
310 While GNOS was used for the core pipelines, Synapse ${ }^{23}$ was used to provide an interface to the

311 files generated by the working groups and other intermediate results created throughout the project.

312 Unlike GNOS which is focused on archival storage, Synapse allowed for collective editing in the

313 form of a wiki, provenance tracking and versioning of results through a web interface as well as

314 programmatic APIs. While Synapse provided an interface that allowed analyses to be shared

315 rapidly across the consortia, the controlled access data was stored on a secure SFTP server

316 provided by the National Cancer Institute (NCI). When the working groups complete their

317 analysis, the metadata is retained in Synapse while the final version of the results is transferred to

318 the ICGC Data Portal for archival.

319 In addition to GNOS-based repositories, the PCAWG dataset has been mirrored to multiple

320 locations: the European Archive (EGA,

321 https://www.ebi.ac.uk/ega/studies/EGAS00001001692), AWS Simple Storage Service (S3,

322 https://dcc.icgc.org/icgc-in-the-cloud/aws), and the Cancer Genome Collaboratory

323 (http://cancercollaboratory.org). The data holdings at each repository at the time of publication are

324 summarized in Suppl Table 2. To help researchers locate the PCAWG data, the ICGC Data Portal

325 (https://dcc.icgc.org) provides a faceted search interface to query about donor, cancer type, data

326 type or data repositories. Users can browse the collection of released PCAWG data and generate

327 a manifest that facilitates downloading of the selected files.

328 The data repositories hosted at AWS S3 and the Collaboratory are powered by an open source

329 object-based ICGC Storage System (https://github.com/icgc-dcc/dcc-storage) that enables fast,

330 secure and multi-part downloads of files. Since AWS and the Collaboratory also have compute

331 power co-located with the PCAWG data, they serve as effective cloud resources for researchers 
332 wishing to conduct further analyses on the PCAWG data without having to provision local

333 compute resources and to download terabytes of data to their local compute environment.

\section{Discussion: Replicating PCAWG Analysis on Your Own Data}

335 This project provided us with a rare opportunity to directly compare three categories of compute

336 environment: traditional HPC, academic compute clouds and commercial clouds. In terms of

337 stability and first time setup effort, we found that the traditional HPC environment routinely

338 outperformed academic cloud systems, and often outperformed the commercial clouds. However,

339 most of the academic cloud systems we worked with had been recently installed and some of the

340 stability issues resulted from the shake-down period. The major benefit of the commercial clouds

341 was the ability to scale compute resources up or down as needed, the ease of replicating the setup

342 in different regions, and the availability of cloud-based data centers in different geographic

343 regions, which allowed us to minimize data transfer overhead. For groups interested in replicating

344 PCAWG results, or using the analytic pipelines for their own data, we are comfortable

345 recommending running the analysis on a commercial cloud.

346 In terms of cost, we have summarized in Figure 5 the costs of computing on AWS and the tradeoff

347 in accuracy if running a subset of the variant calling pipelines. The cost of aligning one normal

348 specimen and one tumor specimen, and running three variant calling workflows followed by the

349 OxoG workflow is about $\$ 100$ per donor. This is based on a mean WGS coverage of $30 \mathrm{X}$ for

350 normal specimens, and a bimodal coverage distribution with maxima at $38 \mathrm{X}$ and $60 \mathrm{X}$ for tumor

351 specimens ${ }^{24}$. In addition, the hourly rate of the VMs are approximated from the spot instance

352 pricing we experienced during production runs. With three variant calling workflows, we achieved

353 an F1 score of 0.92 . If one is willing to sacrifice some accuracy in order to reduce costs, then 
354 running only one variant calling workflow may be an option. Despite the higher costs, running two

355 workflows does not result in increased accuracy. Unfortunately, we were not able to directly

356 compare the analysis costs among commercial clouds, academic clouds and HPC due to the

357 difficulty in assessing the fully loaded cost of provisioning and running an academic compute

358 cluster.

359 In terms of time, the major benefit of operating on commercial clouds is the availability of ample

360 resources for simultaneous parallel runs. For example, in a scenario to analyze a total of 100

361 donors, one runs 200 VMs each aligning one tumor or normal specimen, followed by 300 VMs

362 each running one of the three variant calling workflows on one donor, and 100 VMs to run OxoG

363 workflow, the analysis will in principle take under 9 days to complete. In practice, additional time

364 must be allowed for testing, scaling up, and the inevitability of failed jobs. A more realistic

365 estimate of the time taken to run 100 donors through the complete PCAWG analysis on a

366 commercial cloud is a few weeks.

367 Another issue when planning a large-scale genome analysis project is the variance in execution

368 time from donor to donor. The variant calling pipelines took between 40 and 65 hours of wall time

369 to complete a tumor/genome pair, with the EMBL/DKFZ pipeline running the quickest and the

370 Broad and Sanger pipelines taking somewhat longer. In addition to the variant calling step, the

371 Broad pipeline was preceded by a GATK co-cleaning process taking an additional 24 hours. For

372 each pipeline there was significant variation in the runtime taken for each genome, and some

373 tumor/normal pairs required an excessive amount of time to complete. Because long-running jobs

374 can have economic and logistic impacts, we investigated the cause of this variation by applying

375 linear regression to a number of features describing the raw sequencing sets, including coverage,

376 read quality and mapping scores, number of mismatched end pairs and others (data not shown). 
377 We found that a single factor, genomic coverage, explained the variation in wall clock time which

378 increased roughly linearly with coverage.

379 In conclusion, we tackled the challenge of performing uniform analysis on a large dataset across a

380 geographically and technologically disparate collection of compute resources by developing

381 technologies that realized the efficiencies of moving algorithms to the data. This is becoming a

382 necessity as genomic datasets continue to increase in size and are geographically distributed with

383 some jurisdictions restricting the geographical storage and computing of specific datasets. Our

384 approach serves as a model for large scale collaborative efforts that engage many organizations

385 and spread the computation work around the globe.

386 Our effort resulted in three key deliverables. First and foremost, we produced a high-quality,

387 validated consensus variant and alignment dataset of 2,834 cancer donors. To date, this is the

388 largest whole genome cancer dataset analyzed in a consistent and uniform way. The dataset formed

389 the basis for the research by the PCAWG working groups, and will continue to provide value to

390 the research community for many years into the future. Second, we produced a series of best-

391 practice analytical workflows that are portable through the use of Docker and are available on the

392 Dockstore. These workflows are usable in a multitude of compute environments giving researchers

393 the ability to replicate our analysis on their own data. Finally, the infrastructure we built to

394 coordinate analyses between cloud and HPC environments will be helpful for other projects

395 requiring the same distributed approaches.

\section{Acknowledgements}

397 The authors would like to acknowledge the donation of the following compute resources: the

398 PRACE Research Infrastructure resource MareNostrum3 at Barcelona Supercomputing Center 
with technical expertise provided by the Red Española de Supercomputación and funding support by the Spanish Ministry of Health, ISCIII, in the project Instituto Nacional de Bioinformática (PRB2: PT13/0001/0028); the Cancer Genome Collaboratory, jointly funded by the Natural

402 Sciences and Engineering Research Council of Canada, the Canadian Institutes of Health 403 Research, Genome Canada, and the Canada Foundation for Innovation, and with in-kind support 404 from the Ontario Research Fund of the Ministry of Research, Innovation and Science through the 405 Discovery Frontiers: Advancing Big Data Science in Genomics Research program (grant no. RGPGR/448167-2013); the EMBL-EBI Embassy Cloud supported by UK's (BBSRC) Large

407 Facilities Capital Fund and Cancer Research UK's EMBL-EBI Bioinformatics Resource (grant no. C32939/A20952); sFTP server provided by the Center for Biomedical Informatics \&

409 Information Technology (CBIIT) at National Cancer Institute; infrastructure at the Ontario 410 Institute for Cancer Research funded by the Government of Ontario and the Canada Foundation 411 for Innovation (Project \#21039); ETRI's OpenStack supported by Institute for Information \& 412 communications Technology Promotion with funding from the Korea government (MSIP) 413 (No.B0101-15-0104, The Development of Supercomputing System for the Genome Analysis), 414 Ministry of Health \& Welfare, Republic of Korea (grant no: HI14C0072), Korean national research 415 foundation (grant no NRF-2017R1A2B2012796, NRF-2016R1D1A1B03934110), and generous 416 support from Wan Choi and Kwang-Sung; 'Shirokane'_provided by Human Genome Center, the 417 Institute of Medical Science, the University of Tokyo along with technical assistance from Hitachi, 418 Ltd.; Microsoft Azure contributed through a grant to the UC Santa Cruz Genomics Institute and 419 supported by the National Human Genome Research Institute of the National Institutes of Health 420 (grant no U54HG007990) and NCI ITCR (grant no 1R01CA180778); iDASH HIPAA cloud which 
421 is a member of the NIH/NHLBI National Centers for Biomedical Computing (U54HL108460) to

422 UC San Diego Health Sciences, Department of Biomedical Informatics.

423 In addition, the Broad team was supported by G.G. funds at MGH and Broad Institute. The DKFZ

424 team was supported by the BMBF-funded Heidelberg Center for Human Bioinformatics (HD-

425 HuB) within the German Network for Bioinformatics Infrastructure (de.NBI) (\#031A537A,

426 \#031A537C) and the BMBF-funded grants ICGC PedBrain (01KU1201A, 01KU1201B), ICGC

427 EOPC (01KU1001A), ICGC MMML-seq (01KU1002B), and ICGC DE-MINING (01KU1505E).

428 Variant calling with the DKFZ/EMBL pipeline made use of the Roddy framework, and provision

429 of data and metadata of the German ICGC projects was assisted by the One Touch Pipeline (OTP).

430 The OICR team was funded by the Government of Ontario and the Canada Foundation for

431 Innovation (Project \#21039). The Sanger team was supported by the Wellcome Trust grant

432 (098051) with contributions by Shriram G Bhosle, David R Jones, Andrew Menzies, Lucy

433 Stebbings, Jon W Teague.

434

435 References

436 1. Network, T.C.G.A.R. et al. The Cancer Genome Atlas Pan-Cancer analysis project. Nature 437 Genetics 45, 1113-1120 (2013).

438 2. PCAWG-3. Pan-Cancer Study of Recurrent and Heterogeneous RNA Aberrations and 439 Association with Whole-Genome Variants. (in preparation).

440 3. Alioto, T.S. et al. A comprehensive assessment of somatic mutation detection in cancer

441 using whole-genome sequencing. Nat Commun 6, 10001 (2015).

442 4. PCAWG-1. Consistent Detection of Short Somatic Mutations in 2,778 Cancer Whole 443 Genomes. (in preparation). 
444 5. Phillips, M. \& Knoppers, B. Building an International Code of Conduct for Genomic Cloud

445 Research. (in preparation).

446 6. Wilks, C. et al. The Cancer Genomics Hub (CGHub): overcoming cancer through the

447 power of torrential data. Database (Oxford) 2014(2014).

448 7. Li, H. Aligning sequence reads, clone sequences and assembly contigs with BWA-MEM.

449 (2013).

450 8. Jones, D. et al. cgpCaVEManWrapper: Simple Execution of CaVEMan in Order to Detect

451 Somatic Single Nucleotide Variants in NGS Data. Curr Protoc Bioinformatics 56, 15.10.1-

$452 \quad 15.10 .18(2016)$.

453 9. Raine, K.M. et al. cgpPindel: Identifying Somatically Acquired Insertion and Deletion

454 Events from Paired End Sequencing. Curr Protoc Bioinformatics 52, 15.7.1-12 (2015).

455 10. Raine, K.M. et al. ascatNgs: Identifying Somatically Acquired Copy-Number Alterations

456 from Whole-Genome Sequencing Data. Curr Protoc Bioinformatics 56, 15.9.1-15.9.17 (2016).

457 11. BRASS. (https://github.com/cancerit/BRASS).

458 12. Rimmer, A. et al. Integrating mapping-, assembly- and haplotype-based approaches for 459 calling variants in clinical sequencing applications. Nat Genet 46, 912-8 (2014).

460 13. Rausch, T. et al. DELLY: structural variant discovery by integrated paired-end and split461 read analysis. Bioinformatics 28, i333-i339 (2012).

462 14. Cibulskis, K. et al. Sensitive detection of somatic point mutations in impure and 463 heterogeneous cancer samples. Nat Biotechnol 31, 213-9 (2013).

464 15. Fan, Y. et al. MuSE: accounting for tumor heterogeneity using a sample-specific error 465 model improves sensitivity and specificity in mutation calling from sequencing data. Genome Biol $46617,178(2016)$. 
16. Costello, M. et al. Discovery and characterization of artifactual mutations in deep coverage

468

469

470

471

472 (2016).

473

474

475

476

477

478

479

480

481

482

483

484

485

486

487

488

489

490

491

492

targeted capture sequencing data due to oxidative DNA damage during sample preparation.

Nucleic Acids Res 41, e67 (2013).

17. Wala, J., Zhang, C.Z., Meyerson, M. \& Beroukhim, R. VariantBam: filtering and profiling

of next-generational sequencing data using region-specific rules. Bioinformatics 32, 2029-31

18. PCAWG-6. PCAWG-6 paper. (in preparation).

19. PCAWG-11. PCAWG-11 paper. (in preparation).

20. O'Connor, B.D., Merriman, B. \& Nelson, S.F. SeqWare Query Engine: storing and searching sequence data in the cloud. BMC Bioinformatics 11 Suppl 12, S2 (2010).

21. O'Connor, B.D. et al. The Dockstore: enabling modular, community-focused sharing of Docker-based genomics tools and workflows. F1000Res 6, 52 (2017).

22. Amstutz, P. et al. Common Workflow Language, v1.0. figshare (2016).

23. Omberg, L. et al. Enabling transparent and collaborative computational analysis of 12 tumor types within The Cancer Genome Atlas. Nat Genet 45, 1121-6 (2013).

24. PCAWG-QC. Framework for quality assessment of whole genome, cancer sequences. (in preparation).

\section{Additional Members of the PCAWG Technical Working Group}

Javier Bartolomé Rodriguez ${ }^{1}$, Keith A. Boroevich ${ }^{2}$, Rich Boyce ${ }^{3}$, Angela N. Brooks ${ }^{4}$, Alex Buchanan $^{5}$, Ivo Buchhalter ${ }^{6,7}$, Niall J. Byrne ${ }^{8}$, Andy Cafferkey ${ }^{9}$, Peter J. Campbell ${ }^{10}$, Zhaohong Chen ${ }^{11}$, Sunghoon $\mathrm{Cho}^{12}$, Wan $\mathrm{Choi}^{13}$, Peter Clapham ${ }^{14}$, Francisco M. De La Vega ${ }^{15,16}$, Jonas Demeulemeester $^{17,18}$, Michelle T. Dow ${ }^{19}$, Lewis J. Dursi ${ }^{8,20}$, Juergen Eils ${ }^{21}$, Claudiu Farcas ${ }^{22}$, Francesco Favero ${ }^{23}$, Nodirjon Fayzullaev ${ }^{8}$, Paul Flicek ${ }^{3}$, Nuno A. Fonseca ${ }^{3}$, Josep L.1. Gelpi ${ }^{24,25}$, Gad Getz $^{26,27}$, Bob Gibson ${ }^{8}$, Michael C. Heinold ${ }^{7,6}$, Julian M. Hess ${ }^{26}$, Oliver Hofmann ${ }^{28}$, Jongwhi H. Hong ${ }^{29}$, Thomas J. Hudson ${ }^{30,31}$, Daniel Huebschmann ${ }^{6,7}$, Barbara Hutter ${ }^{32,33}$, Carolyn M. 
Hutter $^{34}$, Seiya Imoto ${ }^{35}$, Sinisa Ivkovic ${ }^{36}$, Seung-Hyup Jeon ${ }^{13}$, Wei Jiao ${ }^{8}$, Jongsun Jung ${ }^{37}$, Rolf Kabbe $^{6}$, Andre Kahles ${ }^{38,39}$, Jules Kerssemakers ${ }^{40}$, Hyunghwan Kim ${ }^{13}$, Hyung-Lae Kim ${ }^{41,42}$, Jihoon Kim ${ }^{11}$, Jan O. Korbel ${ }^{43,3}$, Michael Koscher ${ }^{40}$, Antonios Koures ${ }^{11}$, Milena Kovacevic ${ }^{36}$, Chris Lawerenz ${ }^{6}$, Ignaty Leshchiner ${ }^{26}$, Dimitri G. Livitz ${ }^{26}$, George L. Mihaiescu ${ }^{8}$, Sanja Mijalkovic ${ }^{36}$, Ana Mijalkovic Lazic ${ }^{36}$, Satoru Miyano ${ }^{44}$, Hardeep K. Nahal ${ }^{8}$, Mia Nastic ${ }^{36}$, Jonathan Nicholson ${ }^{14}$, David Ocana $^{3}$, Kazuhiro Ohi ${ }^{44}$, Lucila Ohno-Machado ${ }^{22}$, Larsson Omberg $^{45}$, B.F. Francis Ouellette ${ }^{8,46}$, Nagarajan Paramasivam ${ }^{6,47}$, Marc D. Perry ${ }^{8}$, Todd D. Pihl ${ }^{48}$, Manuel Prinz ${ }^{6}$, Montserrat Puiggròs ${ }^{24}$, Petar Radovic ${ }^{36}$, Esther Rheinbay ${ }^{26,49}$, Mara W. Rosenberg $^{26,49}$, Charles Short $^{3}$, Heidi J. Sofia ${ }^{50}$, Jonathan Spring ${ }^{51}$, Adam J. Struck ${ }^{5}$, Grace Tiao $^{26}$, Nebojsa Tijanic ${ }^{36}$, Peter Van Loo ${ }^{17,18}$, David Vicente ${ }^{1}$, Jeremiah A. Wala ${ }^{26,52}$, Zhining Wang $^{53}$, Johannes Werner $^{6}$, Ashley Williams ${ }^{11}$, Youngchoon Woo $^{13}$, Adam J. Wright ${ }^{8}$, Qian Xiang $^{8}$

${ }^{1}$ Department of Operations, Barcelona Supercomputing Center, Barcelona, Catalunya, 8034, Spain. ${ }^{2}$ Laboratory for Medical Science Mathematics, RIKEN Center for Integrative Medical Sciences, Yokohama, Kanagawa, 230-0045, Japan. ${ }^{3}$ European Molecular Biology Laboratory, European Bioinformatics Institute, Hinxton, Cambridge, CB 10 1SD, United Kingdom. ${ }^{4}$ Biomolecular Engineering, University of California Santa Cruz, Santa Cruz, California, 95065, USA. ${ }^{5}$ Department of Computational Biology, Oregon Health and Science University, Portland, Oregon, 97239, USA. ${ }^{6}$ Division of Theoretical Bioinformatics, German Cancer Research Center (DKFZ), Heidelberg, Baden-Württemberg, 69120, Germany. ${ }^{7}$ Department for Bioinformatics and Functional Genomics, Institute for Pharmacy and Molecular Biotechnology and BioQuant, Heidelberg University, Heidelberg, Baden-Württemberg, 69120, Germany. ${ }^{8}$ Informatics and Biocomputing Program, Ontario Institute for Cancer Research, Toronto, Ontario, M5G 0A3, Canada. ${ }^{9}$ Technical Services Cluster, European Molecular Biology Laboratory, European Bioinformatics Institute, Hinxton, Cambridge, CB10 1SD, United Kingdom. ${ }^{10}$ Cancer Genome Project, Wellcome Trust Sanger Institute, Hinxton, Cambridgeshire, CB10 1SA, United Kingdom ${ }^{11}$ Department of Medicine, University of California San Diego, San Diego, California, 92093, USA. ${ }^{12}$ PDXen Biosystems Inc., Seoul, 4900, South Korea. ${ }^{13}$ Electronics and Telecommunications Research Institute, Daejon, 34129, South Korea. ${ }^{14}$ Informatics Support Group, Wellcome Trust Sanger Institute, Hinxton, Cambridgeshire, CB10 1SA, United Kingdom. ${ }^{15}$ Department of Biomedical Data Science, Stanford University School of Medicine, Stanford, California, 94305, USA. ${ }^{16}$ Annai Systems, Inc., Carlsbad, California, 92011, USA. ${ }^{17}$ The Francis Crick Institute, London, NW1 1AT, United Kingdom. ${ }^{18}$ Department of Human Genetics, University of Leuven, B-3000 Leuven, Belgium ${ }^{19}$ Biomedical Informatics, University of California San Diego, San Diego, California, 92093, USA. ${ }^{20}$ The Centre for Computational Medicine, The Hospital for Sick Children, Toronto, Ontario, M5G 0A4, Canada. ${ }^{21}$ Theoretical Bioinformatics, German Cancer Research Center (DKFZ), Heidelberg, Baden-Württemberg, 69120, Germany. ${ }^{22}$ Health System Department of Biomedical Informatics, University of California San Diego, La Jolla, California, 92093, USA. ${ }^{23}$ BRIC/Finsen Laboratory, Rigshospitalet, Copenhagen, 2200, Denmark. ${ }^{24}$ Department of Life Sciences, Barcelona Supercomputing Center, Barcelona, Catalunya, 8034, Spain. ${ }^{25}$ Department of Biochemistry and Molecular Biomedicine, University of Barcelona, Barcelona, Catalunya, 8028, Spain. ${ }^{26}$ Cancer Program, Broad Institute of MIT and Harvard, Cambridge, Massachusetts, 02142, USA. ${ }^{27}$ Cancer Center and Department of Pathology, Massachusetts General Hospital, Boston, Massachusetts, 02114, USA. ${ }^{28}$ Center for Cancer Research, University of Melbourne, Melbourne, VIC 3001, Australia. ${ }^{29}$ Genome Data Integration Center, Syntekabio Inc., Daejon, 34025, South Korea. ${ }^{30}$ Genomics Program, Ontario Institute for Cancer Research, Toronto, Ontario, M5G 0A3, Canada. ${ }^{31}$ Oncology Discovery and Early Development, AbbVie, Redwood City, California, 94063, USA. ${ }^{32}$ Division of Applied Bioinformatics, German Cancer Research Center (DKFZ), Heidelberg, Baden-Württemberg, 69120, Germany. ${ }^{33}$ Division of Applied Bioinformatics, National Center for Tumor Diseases, Heidelberg, Baden- 
Bethesda, Maryland, 20852, USA. ${ }^{35} \mathrm{Health}$ Intelligence Center, Institute of Medical Science, University of Tokyo, Tokyo, 108-8639, Japan. ${ }^{36}$ Seven Bridges, Cambridge, Massachusetts, 02142, USA. ${ }^{37}$ Genome Data Integration

541 Center, Syntekabio Inc., Daejon, 34025, South Korea ${ }^{38}$ Department of Computer Science, ETH Zurich, Zurich,

542 Zurich, 8092, Switzerland. ${ }^{39}$ Computational Biology Center, Memorial Sloan Kettering Cancer Center, New York,

543 New York, 10065, USA. ${ }^{40}$ German Cancer Research Center (DKFZ), Heidelberg, Baden-Württemberg, 69120,

544 Germany. ${ }^{41}$ Department of Biochemistry, Ewha Womans University, Seoul, O7985, South Korea. ${ }^{42}$ PGM21, Seoul,

545 O7985, South Korea. ${ }^{43}$ Genome Biology Unit, European Molecular Biology Laboratory, Heidelberg, Baden-

546 Württemberg, 69120, Germany. ${ }^{44}$ Human Genome Center, Institute of Medical Science, University of Tokyo,

547 Tokyo, 108-8639, Japan. ${ }^{45}$ Systems Biology, Sage Bionetworks, Seattle, Washington, 98112, USA. ${ }^{46}$ Department of

548 Cell and Systems Biology, University of Toronto, Toronto, Ontario, M5S 3G5, Canada. ${ }^{47}$ Medical Faculty

549 Heidelberg, Heidelberg University, Heidelberg, Baden-Württemberg, 69120, Germany. ${ }^{48}$ CSRA Incorporated,

550 Fairfax, Virginia, 22042, USA. ${ }^{49}$ Cancer Center, Massachusetts General Hospital, Boston, Massachusetts, 02114,

551 USA. ${ }^{50}$ National Human Genome Research Institute, National Institutes of Health, Bethesda, Maryland, 20892-

552 9305, USA. ${ }^{51}$ Center for Data Intensive Science, University of Chicago, Chicago, Illinois, 60637, USA.

$553 \quad{ }^{52}$ Department of Cancer Biology, Dana-Farber Cancer Institute, Boston, Massachusetts, 02115, USA. ${ }^{53}$ TCGA

554 Program Office, National Cancer Institute, Bethesda, Maryland, 20892, USA. 


\section{Figures}

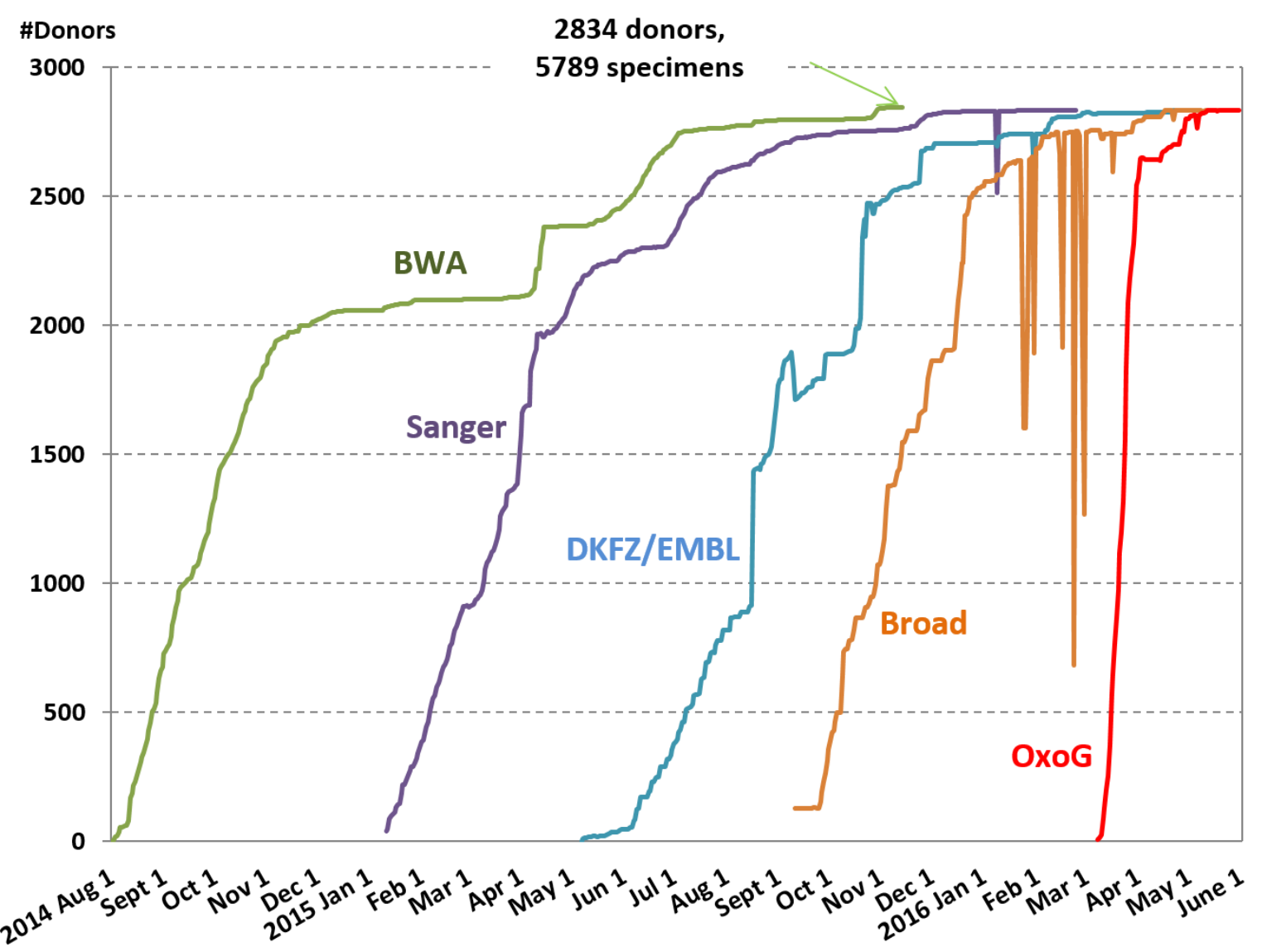

Figure 1: Progress of the 5 workflows over time. The "flat line" of the BWA workflow was due to two major tranches of sequencing data submissions, with a first tranche of $\sim 2000$ donors and a second tranche of $\sim 800$ donors that were uploaded later. The staggered start of the three variant calling pipelines was dictated more by the time required to develop and package the workflows, and less by the availability of compute power. The "dips" on the plots resulted from quality issues with some sets of variant calls that were withdrawn, reprocessed and resubmitted. In the case of the Broad workflow, the variant calls were withdrawn for post-processing before being considered complete. If all workflows and data would have been in place at the beginning of the project, we estimate the computation across the full set of 5,789 genomes could have been completed in under 6 months. 


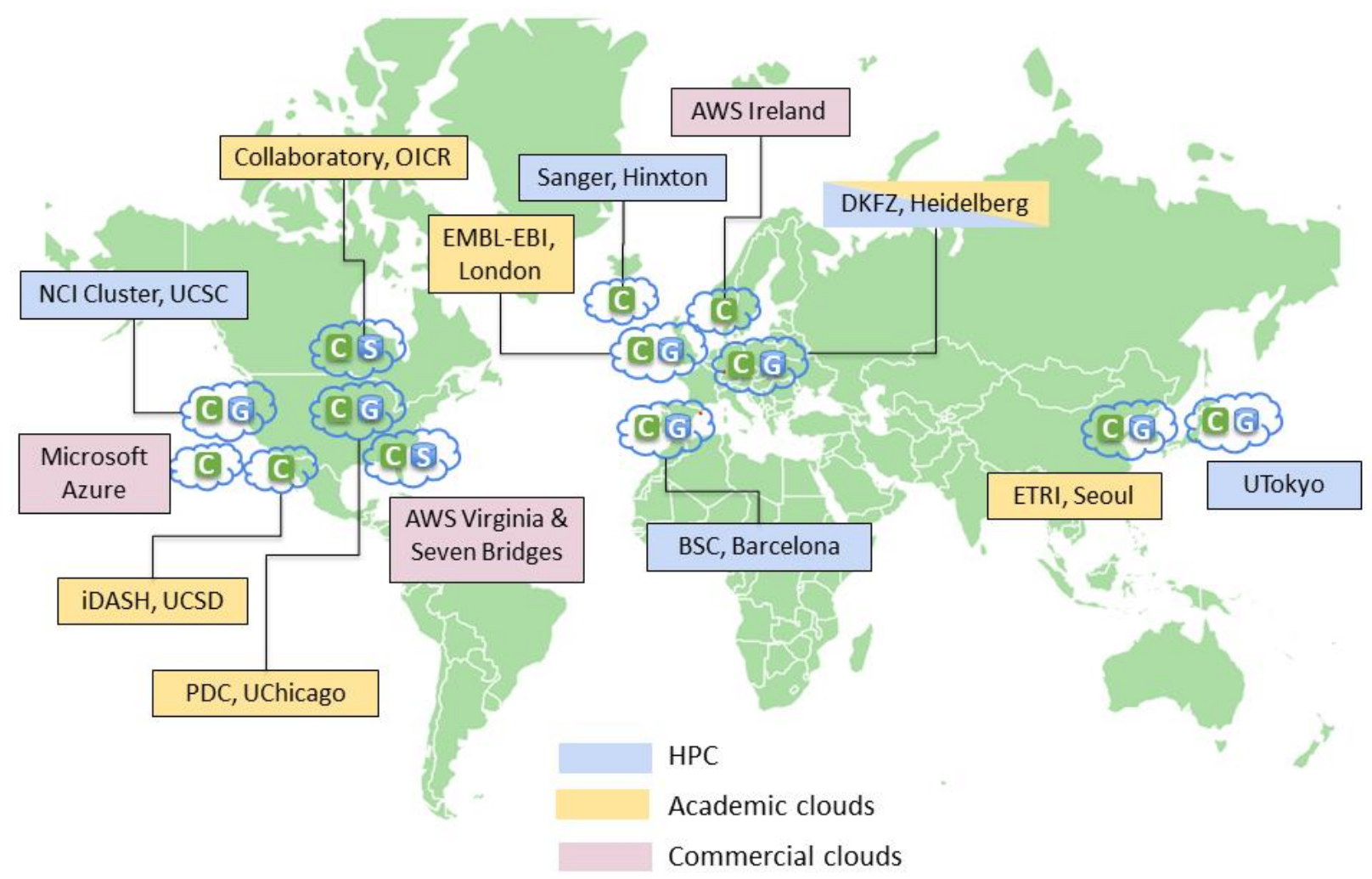


WG BAMs aligned with various aligner \& metadata specific to individual sequencing centers

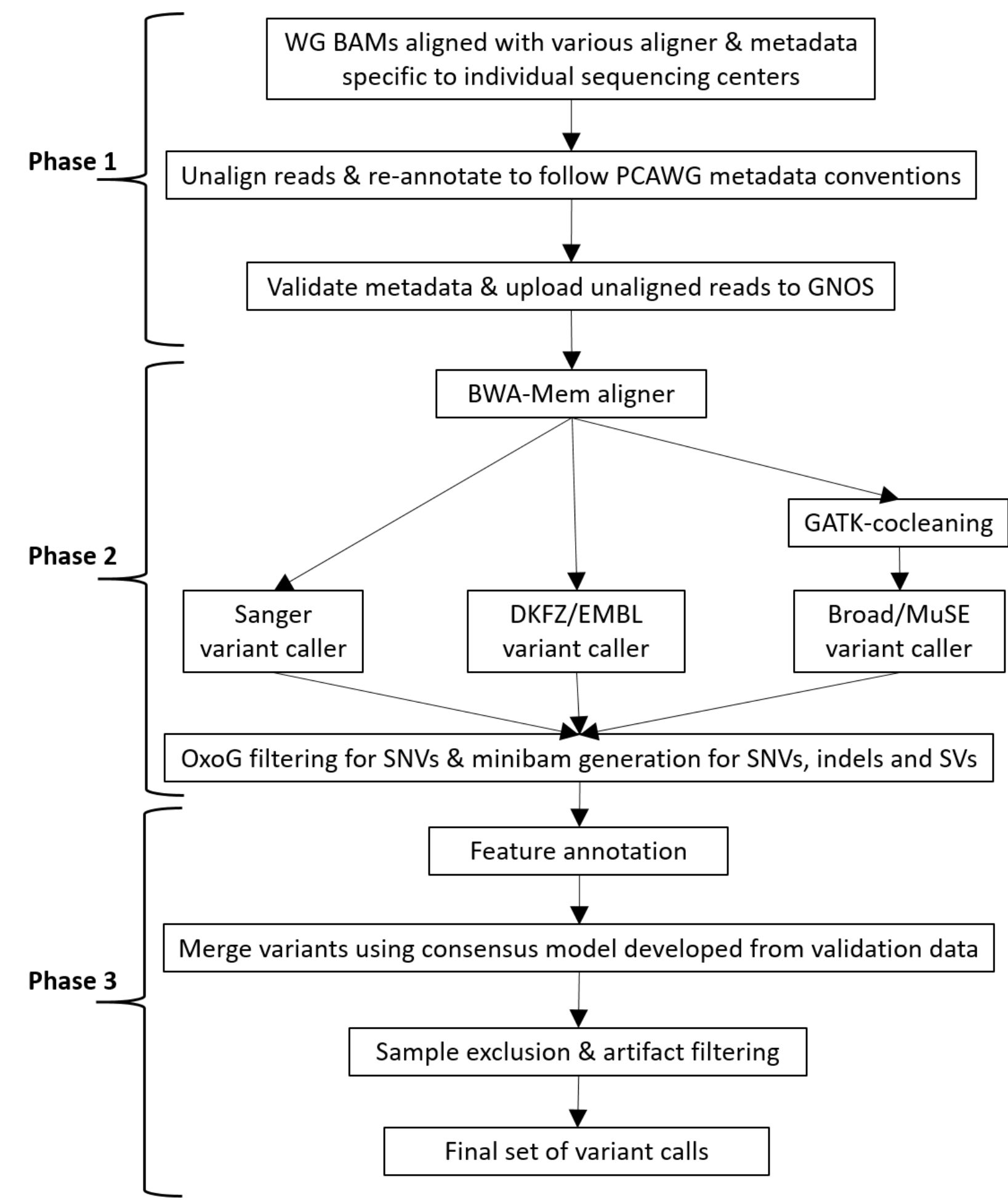

Figure 3: The uniform analysis of whole genomes involves three broad phases. Phase 1: Data marshalling and upload. Phase 2: Sequence alignment and variant calling. Phase 3: Variant merging and filtering. The algorithms for merging SNVs and indels are described in the 579 


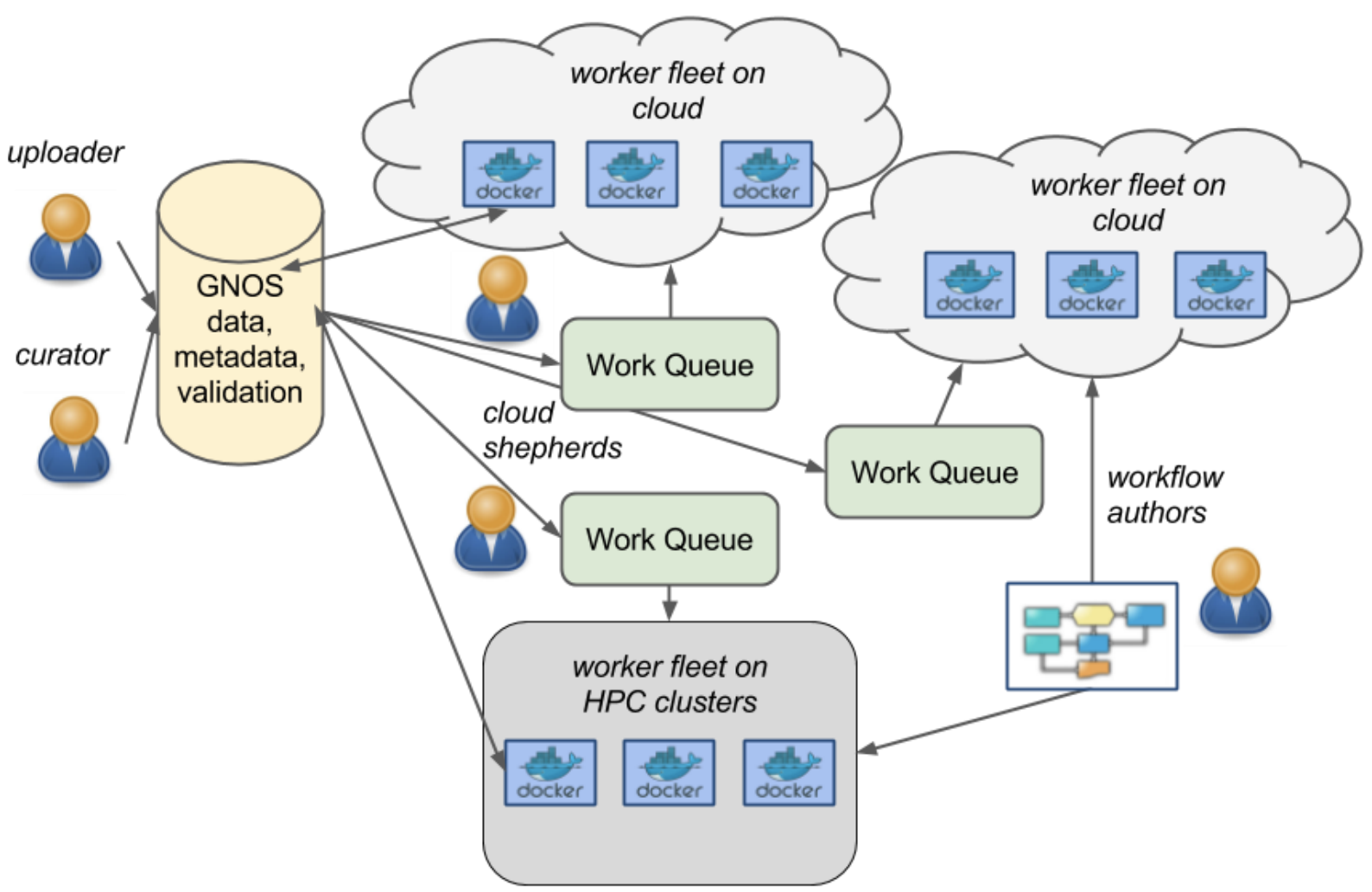

Figure 4: Infrastructure used on cloud and HPC compute environments for core analysis. 


\section{F1 score on SNVs}

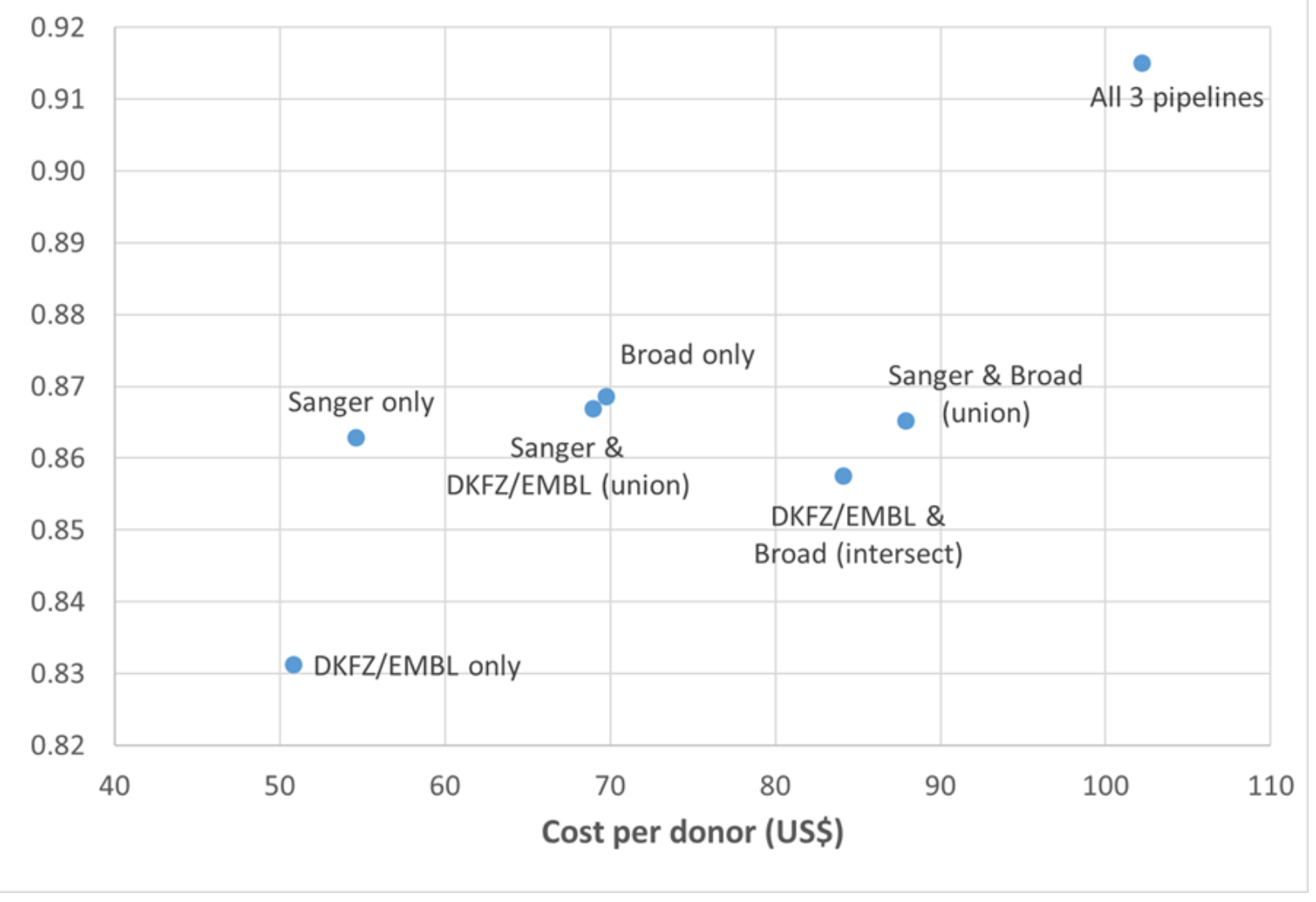

Figure 5: Costs for analyzing a tumor/normal pair through BWA-Mem, different combinations of variant calling pipelines, and OxoG filtering. The cost is calculated based on AWS instances at average spot pricing we experienced during the project, and includes egress costs to transfer the result files. PCAWG ran all 3 variant calling pipelines and achieved an F1 score of 0.9151 for SNVs. If running only one or two pipelines, there will be savings in cost but sacrifice in accuracy. Detailed cost analysis is shown in Suppl Table 3. 
592

593

594

595

596

Table 1. Compute resources. * Shared between environments. ${ }^{* *}$ Transient storage used for local data processing.

\begin{tabular}{|c|c|c|c|c|c|}
\hline & Type & $\begin{array}{l}\text { Allocated } \\
\text { CPU/Cores }\end{array}$ & $\begin{array}{c}\text { Allocated } \\
\text { memory }\end{array}$ & $\begin{array}{l}\text { Data Co-location } \\
\text { Repository }\end{array}$ & $\begin{array}{l}\text { Local Storage } \\
\text { Amount }\end{array}$ \\
\hline AWS & Cloud & variable & variable & $Y$ & 420TB \\
\hline Azure & Cloud & variable & variable & $\mathrm{N}$ & - \\
\hline BSC & HPC & 1000 & 7.75TB & $\mathrm{Y}$ & 300TB \\
\hline Collaboratory & Cloud & 350 & 3.2TB & $Y$ & 132ТВ \\
\hline DKFZ & HPC & 800 & 3.5TB & $\mathrm{Y}$ & 1.7PB ${ }^{*}$ \\
\hline DKFZ & Cloud & 1024 & 4TB & $Y$ & $1.7 \mathrm{~PB}^{*}$ \\
\hline EMBL-EBI & Cloud & 1000 & 4TB & $\mathrm{Y}$ & $1 \mathrm{~PB}$ \\
\hline ETRI & Cloud & 800 & $2 \mathrm{~TB}$ & $\mathrm{Y}$ & 750TВ \\
\hline iDASH & Cloud & 304 & 2.8TB & $\mathrm{N}$ & $9 \mathrm{~TB}^{* *}$ \\
\hline PDC & Cloud & 108 & $324 G B$ & $\mathrm{Y}$ & 732TB \\
\hline Sanger & HPC & 1500 & 12 TB & $\mathrm{N}$ & $750 \mathrm{~TB}^{\star *}$ \\
\hline SBG & Cloud & variable & variable & $Y$ & - \\
\hline UCSC & HPC & 4000 & 33ТВ & $Y$ & 300ТВ \\
\hline UTokyo & HPC & 2496 & 2.5TB & $\mathrm{Y}$ & 400TB \\
\hline
\end{tabular}


598 Table 2. The five core workflows. Components for calling (1) SNVs, (2) indels, (3) SVs and (4) 599 SCNAs in each of the three variant calling workflows are listed. Because we utilized a large 600 number of compute environments with various configurations of cores and RAM, the average 601 runtime for each pipelines varied with large standard deviations (Suppl Fig. 7-10). The runtime 602 for the Broad pipeline included the 24 hours required to run GATK co-cleaning of BAMs. The 603 measured runtime included time to download input files, but not the time to upload result files. 604 (\#) MuSE was developed at MD Anderson Cancer Center and Baylor College of Medicine.

\begin{tabular}{|c|c|c|c|c|c|}
\hline & BWA & Sanger & DKFZ/EMBL & Broad & OxoG \\
\hline $\begin{array}{l}\text { Analytical } \\
\text { components in } \\
\text { workflow }\end{array}$ & $\begin{array}{l}\text { BWA-Mem } \\
\text { Picard } \\
\text { Biobambam } \\
\text { samtools }\end{array}$ & $\begin{array}{l}\text { CaVEMan }^{1} \\
\text { cgpPindel }^{2} \\
\text { BRASS }^{3} \\
\text { ascatNgs }^{4}\end{array}$ & $\begin{array}{c}\text { dkfz_snv }{ }^{1} \\
\text { Platypus }^{2} \\
\text { DELLY3 } \\
\text { ACE-seq }\end{array}$ & $\begin{array}{c}\text { GATK cocleaning } \\
\text { MuTect }^{1} \\
\text { MuSE }^{1, \#} \\
\text { Snowman }^{2,3} \\
\text { dRanger }^{3}\end{array}$ & $\begin{array}{c}\text { OxoG } \\
\text { VariantBam }\end{array}$ \\
\hline Workflow controller & SeqWare & SeqWare & $\begin{array}{l}\text { Roddy, } \\
\text { SeqWare }\end{array}$ & Galaxy & SeqWare \\
\hline $\begin{array}{l}\text { Recommended } \\
\text { compute } \\
\text { requirements }\end{array}$ & $\begin{array}{l}4 \text { cores, } \\
\text { 15GB RAM }\end{array}$ & $\begin{array}{l}16 \text { cores, } \\
4.5 \mathrm{~GB} \\
\text { RAM/core }\end{array}$ & $\begin{array}{l}16 \text { cores, } \\
\text { 64GB RAM }\end{array}$ & $\begin{array}{l}32 \text { cores, } \\
244 \text { GB RAM }\end{array}$ & $\begin{array}{l}8 \text { cores, } \\
\text { 64GB RAM }\end{array}$ \\
\hline $\begin{array}{l}\text { Average runtime } \\
\text { across all compute } \\
\text { environments }\end{array}$ & $\begin{array}{c}2.0+/-1.7 \\
\text { days }\end{array}$ & $\begin{array}{l}5.3+/-5.5 \\
\text { days }\end{array}$ & $\begin{array}{c}3.2+/-1.7 \\
\text { days }\end{array}$ & $\begin{array}{c}5.1+/-2.2 \\
\text { days }\end{array}$ & $\begin{array}{l}2.6+/-1.3 \\
\text { hours }\end{array}$ \\
\hline $\begin{array}{l}\text { Benchmark on } \\
\text { AWS }\end{array}$ & $\begin{array}{l}5.8 \text { days on } \\
\text { 4-core } \\
\text { m1.xlarge }\end{array}$ & $\begin{array}{l}2.2 \text { days on } \\
\text { 32-core } \\
\text { r3.8xlarge }\end{array}$ & $\begin{array}{l}1.7 \text { days on } \\
\text { 32-core } \\
\text { r3.8xlarge }\end{array}$ & $\begin{array}{l}3.7 \text { days on } \\
\text { 32-core r3.8xlarge }\end{array}$ & $\begin{array}{l}4 \text { hours on } \\
\text { 8-core } \\
\text { m2.4xlarge }\end{array}$ \\
\hline Core hours per run & 557 & 1690 & 1306 & 2842 & 32 \\
\hline Output files per run & $120 \mathrm{~GB}$ & $2 \mathrm{~GB}$ & $5 \mathrm{~GB}$ & $35 \mathrm{~GB}$ & $1.5 \mathrm{~GB}$ \\
\hline
\end{tabular}




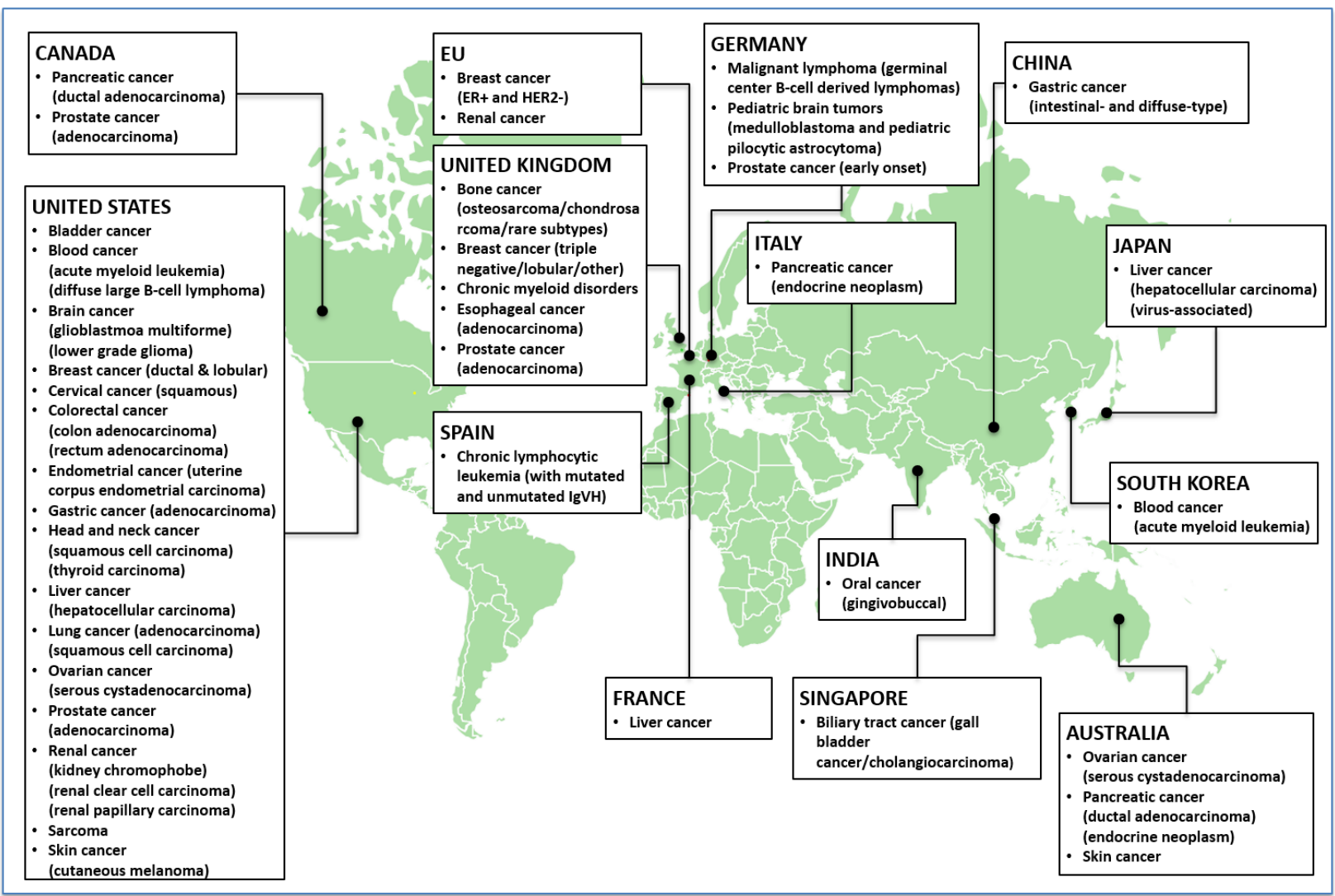
collected from 48 ICGC and TCGA projects in 14 jurisdictions. 


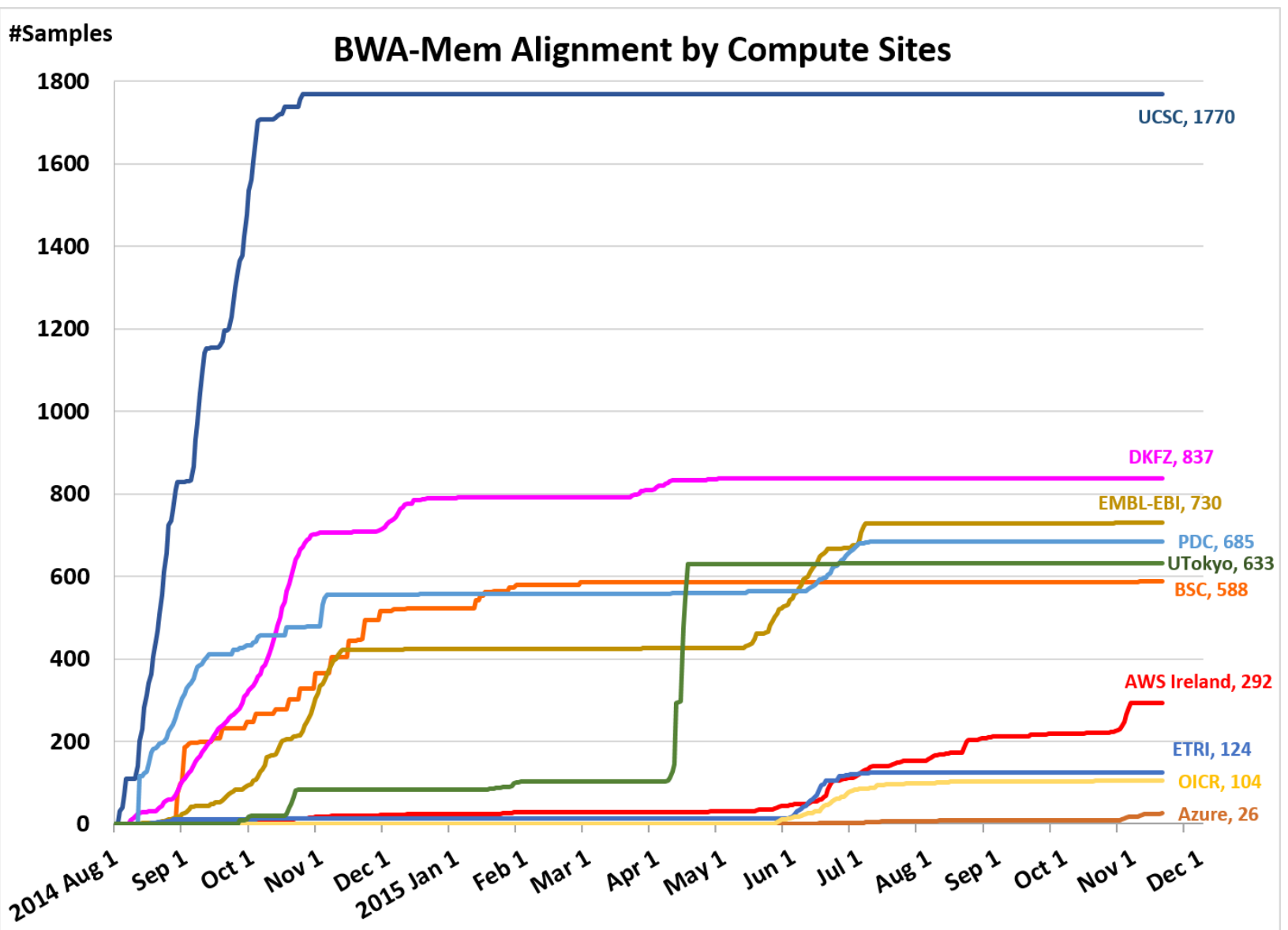

615 616 


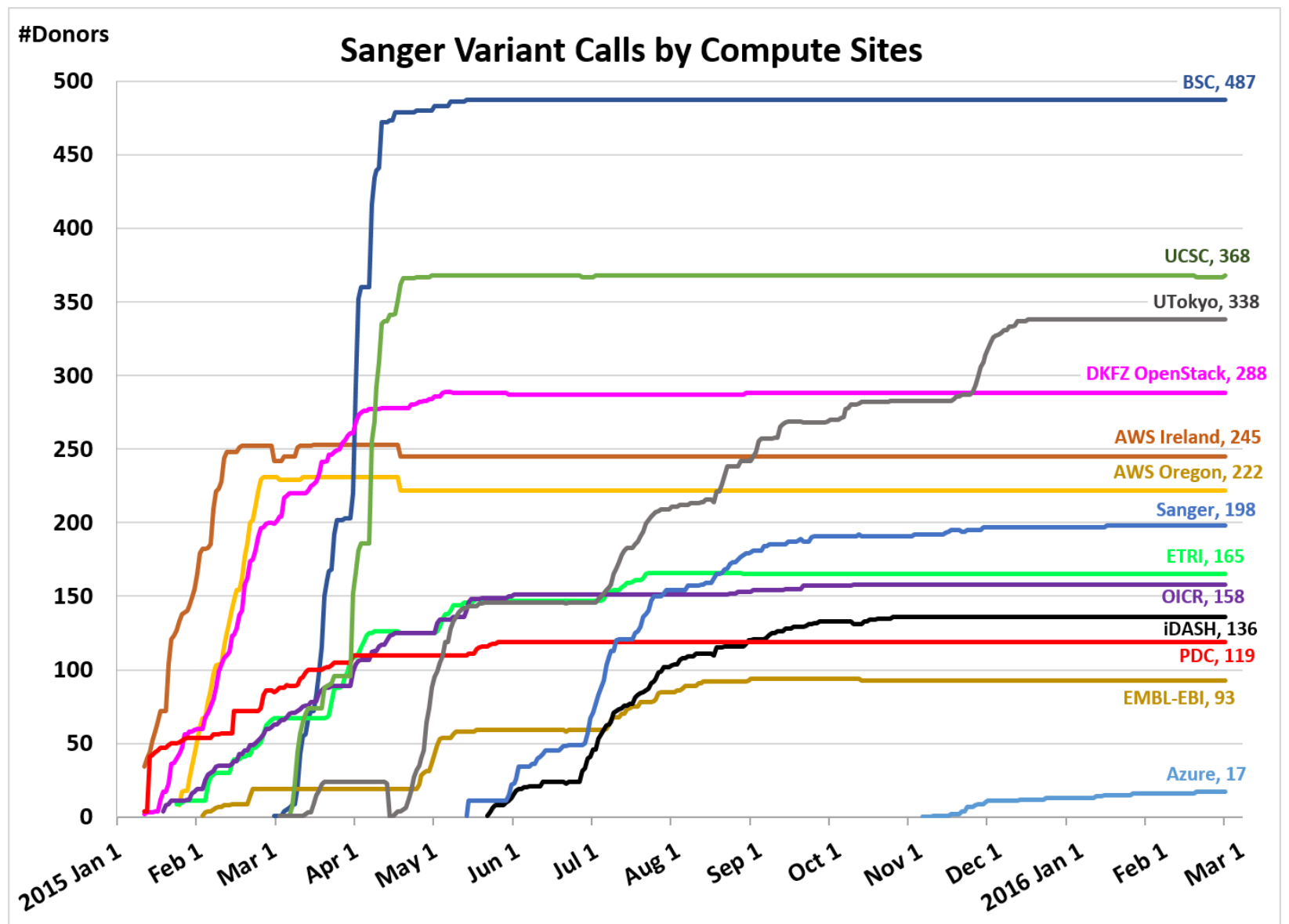

618 Supplementary Figure 3: Progress of Sanger variant calling workflow over time at 13 compute 619 sites. 


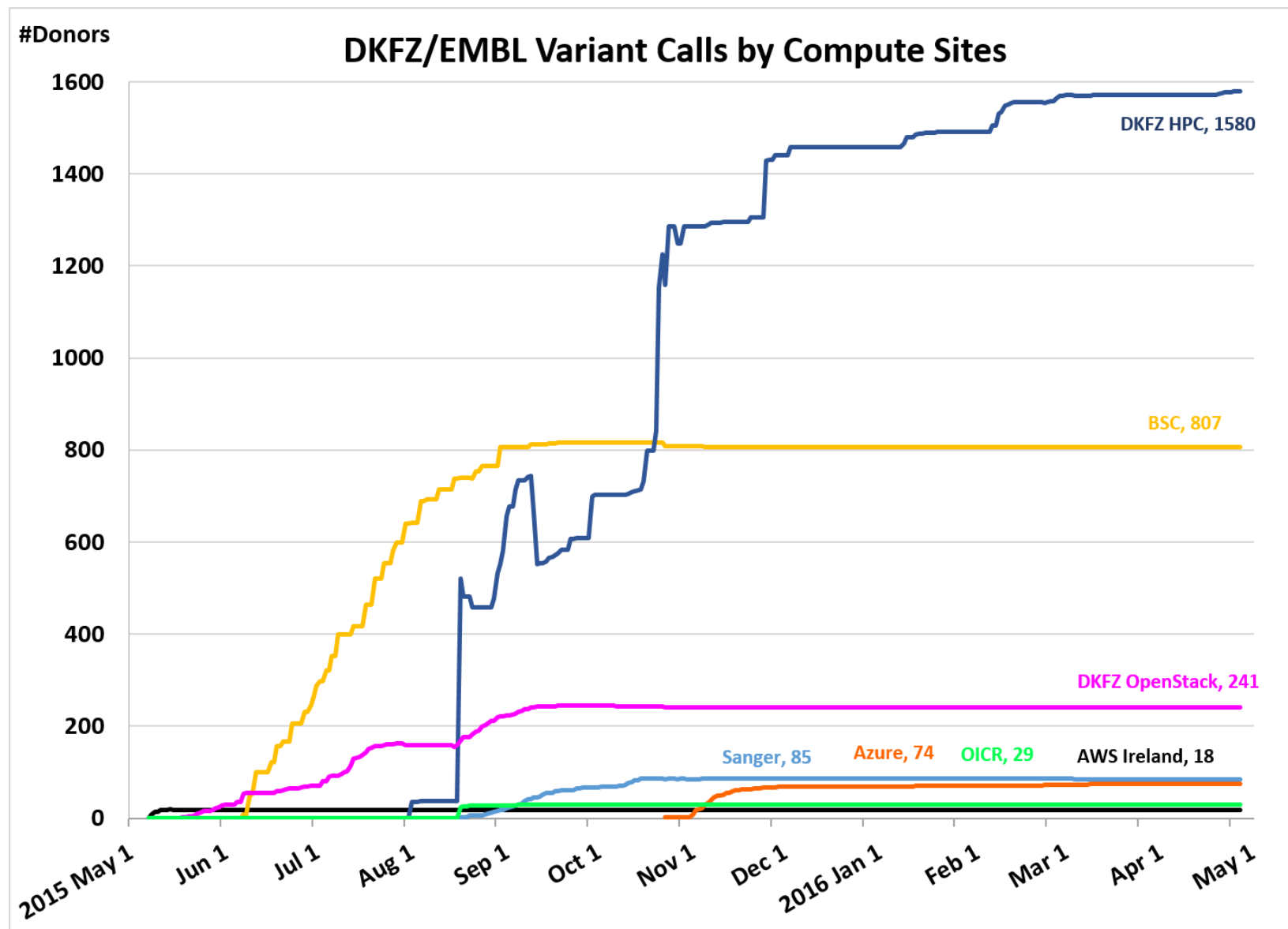
compute sites. 


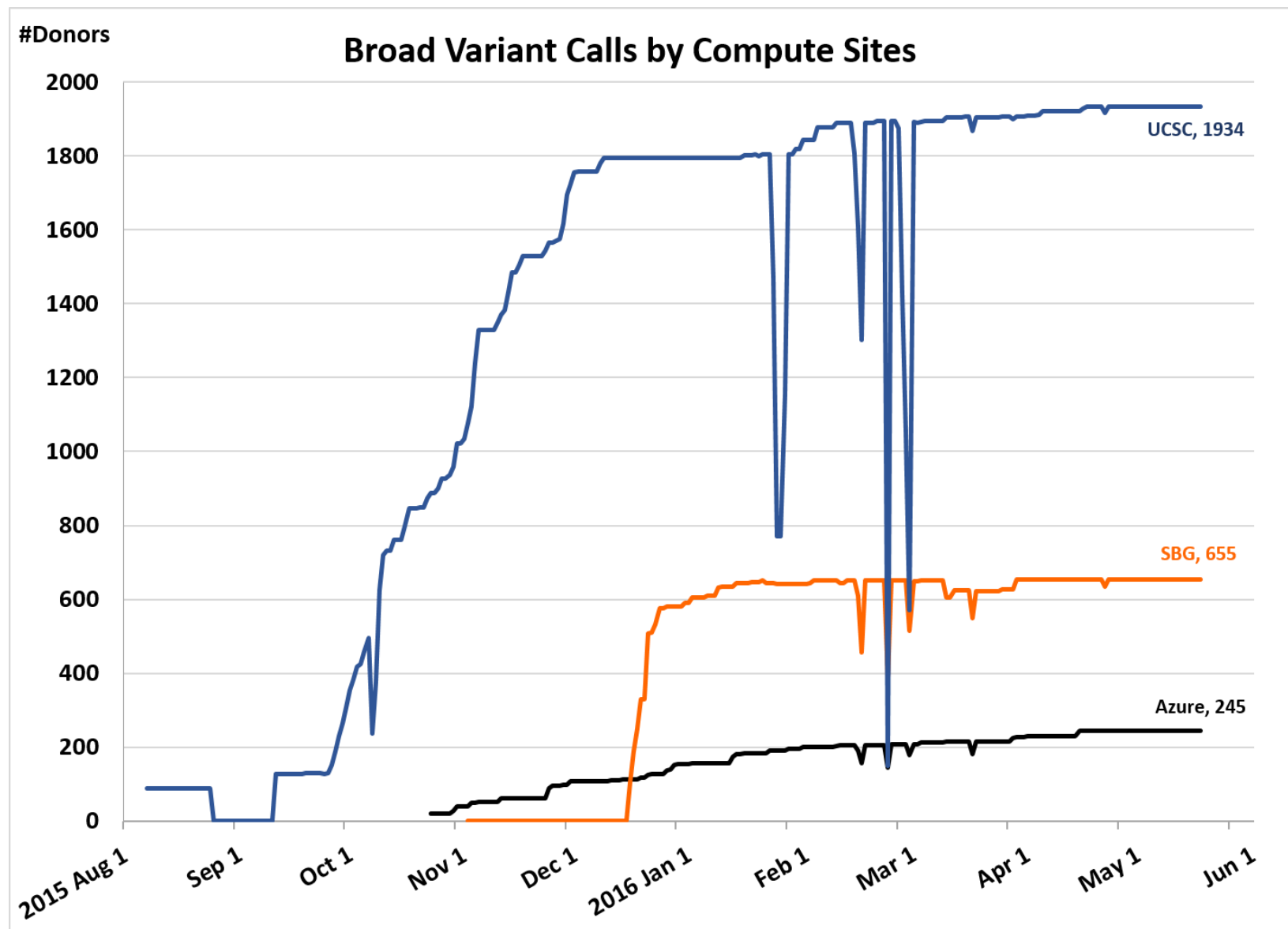
sites. 


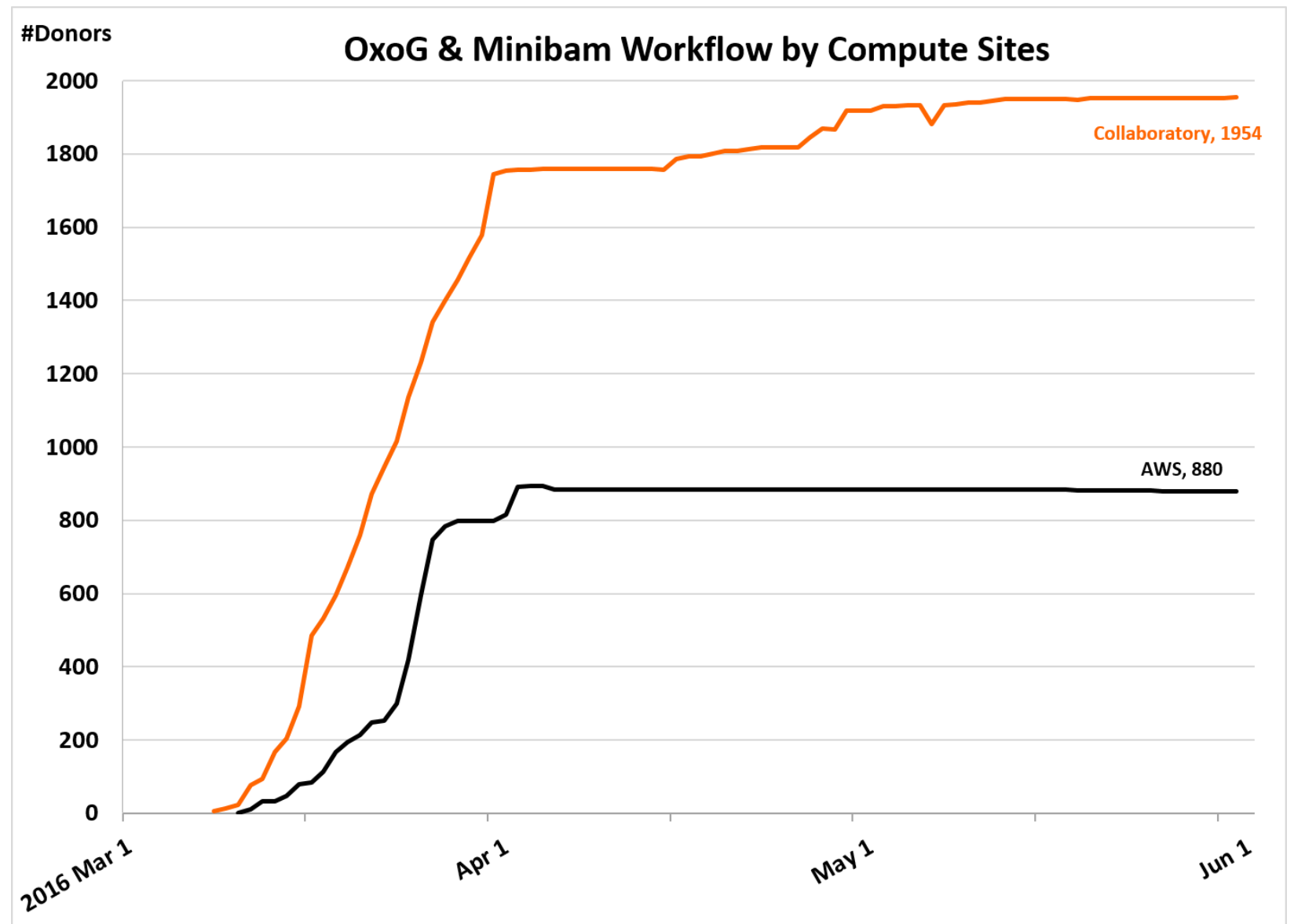

630 Supplementary Figure 6: Progress of OxoG and minibam workflow over time at 2 compute sites. 


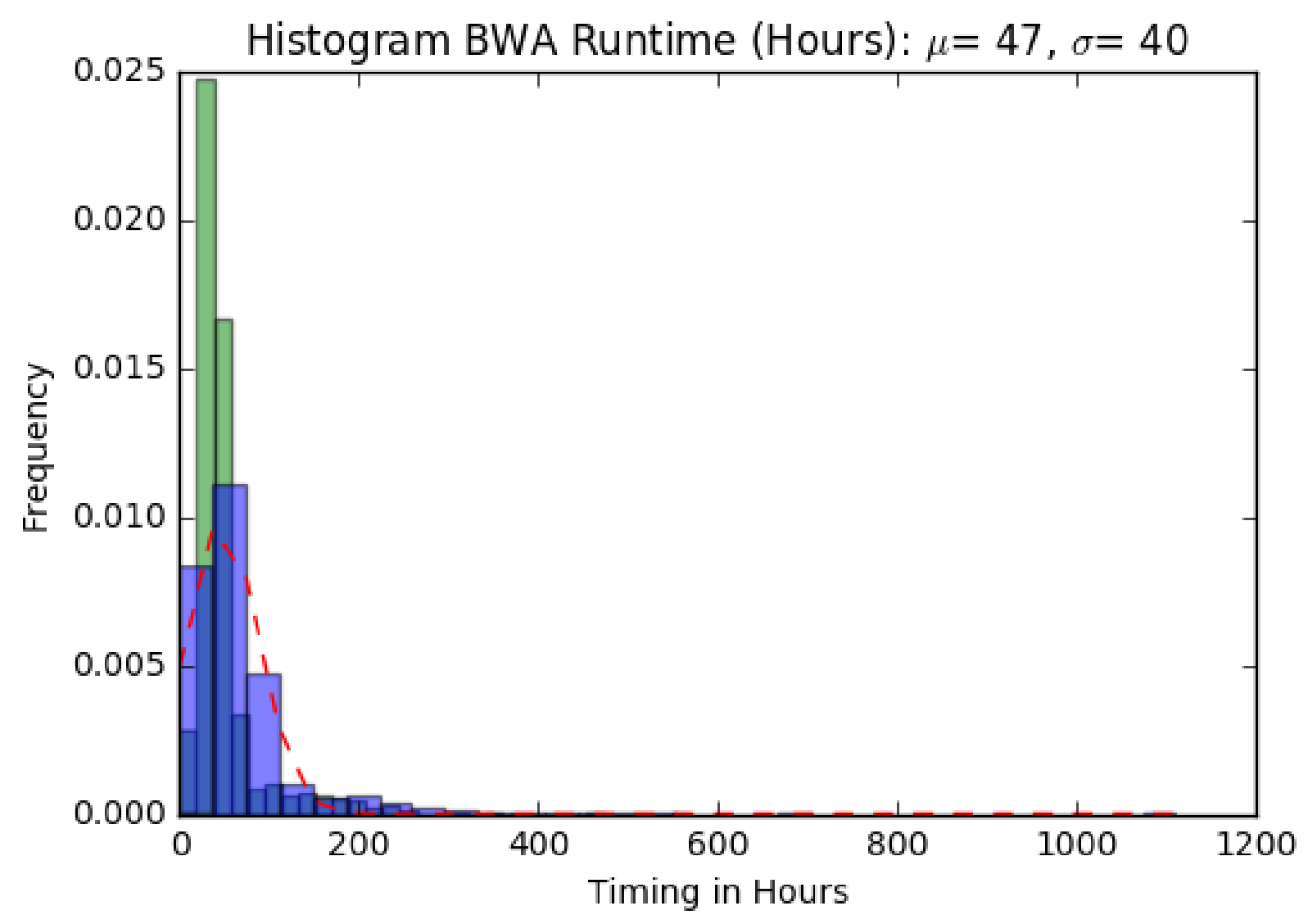

632

633

Supplementary Figure 7: Average runtimes for BWA-Mem alignment workflow

634 


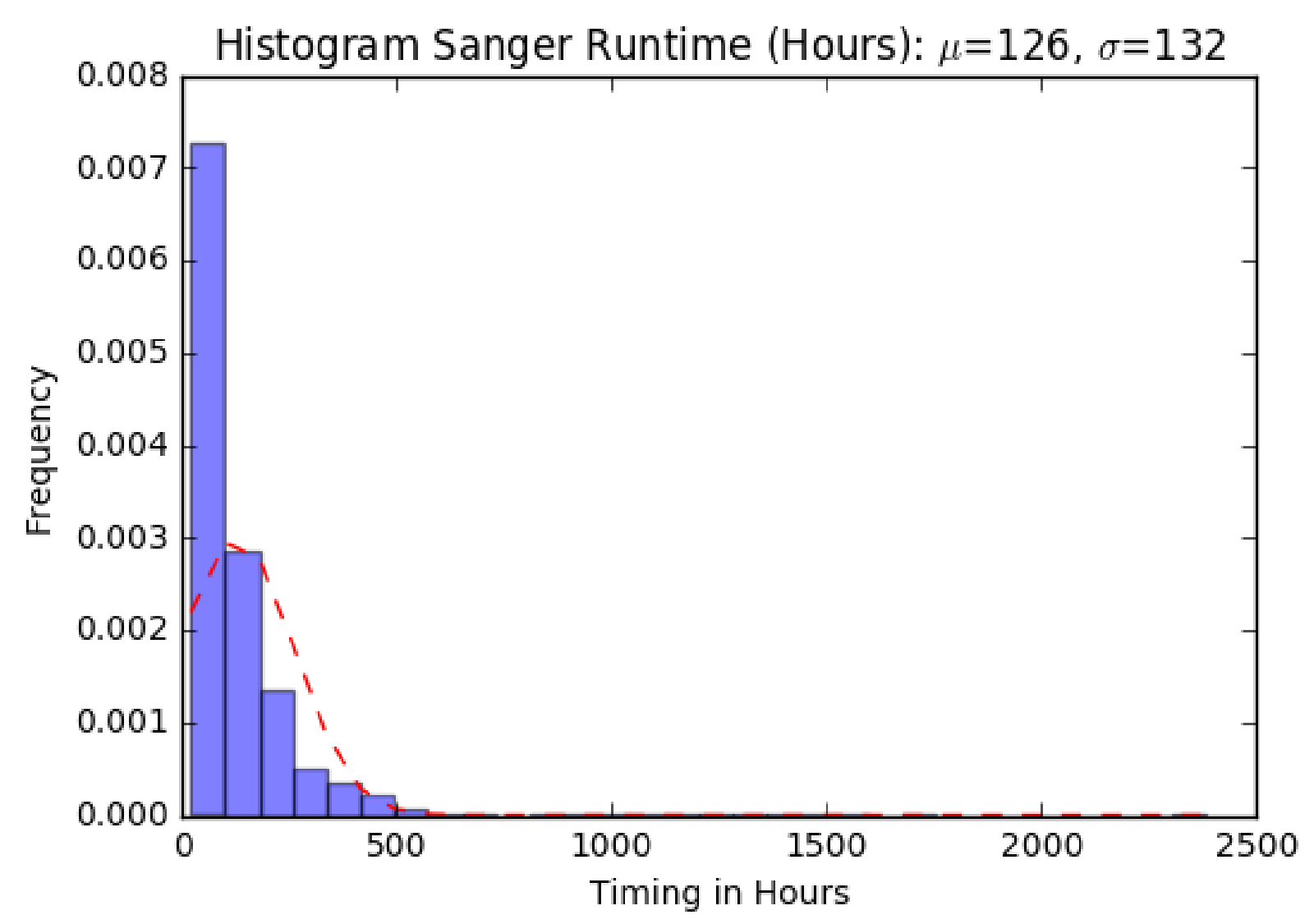




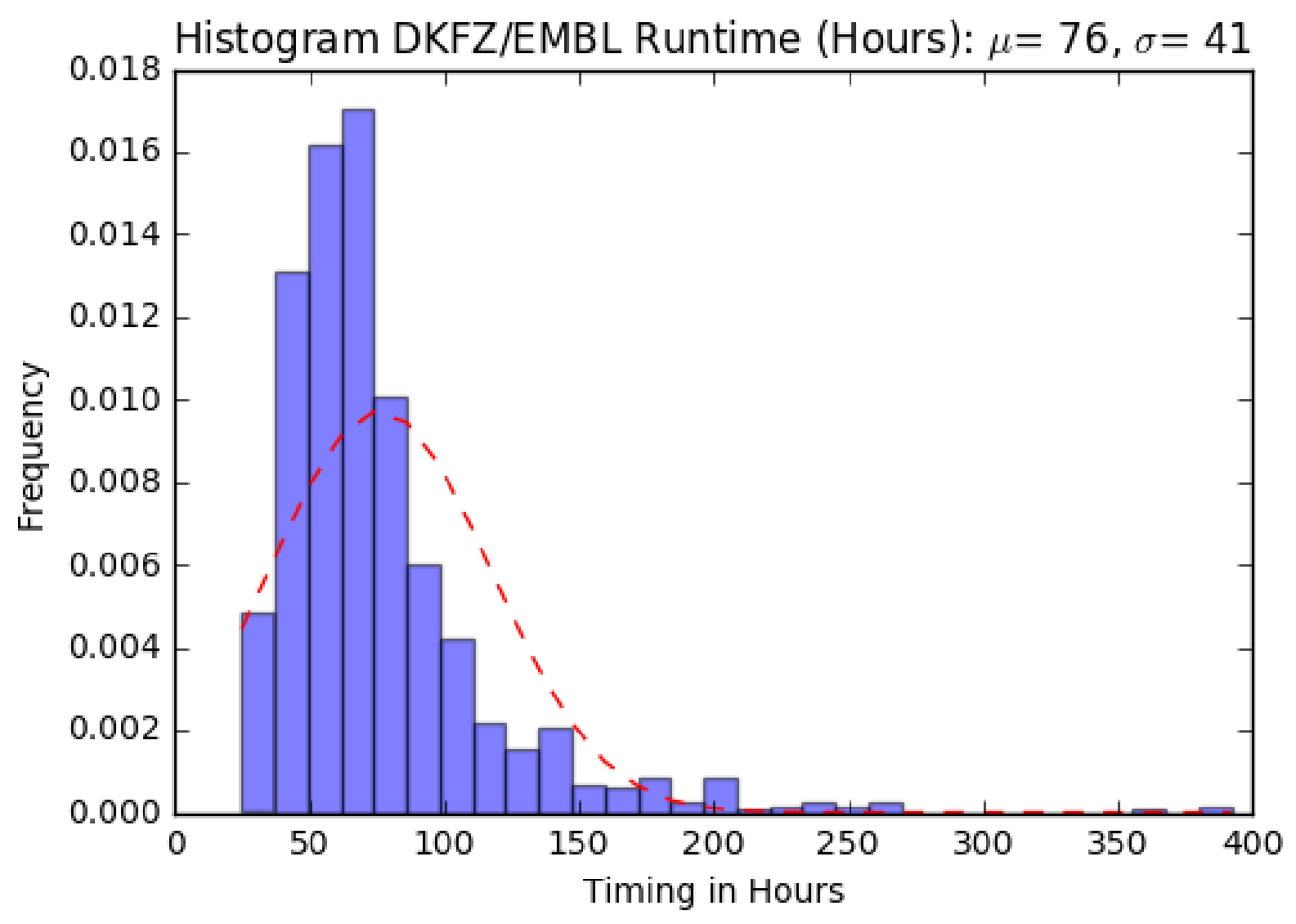

Supplementary Figure 9: Average runtime for the DKFZ/EMBL somatic variant calling workflow. 


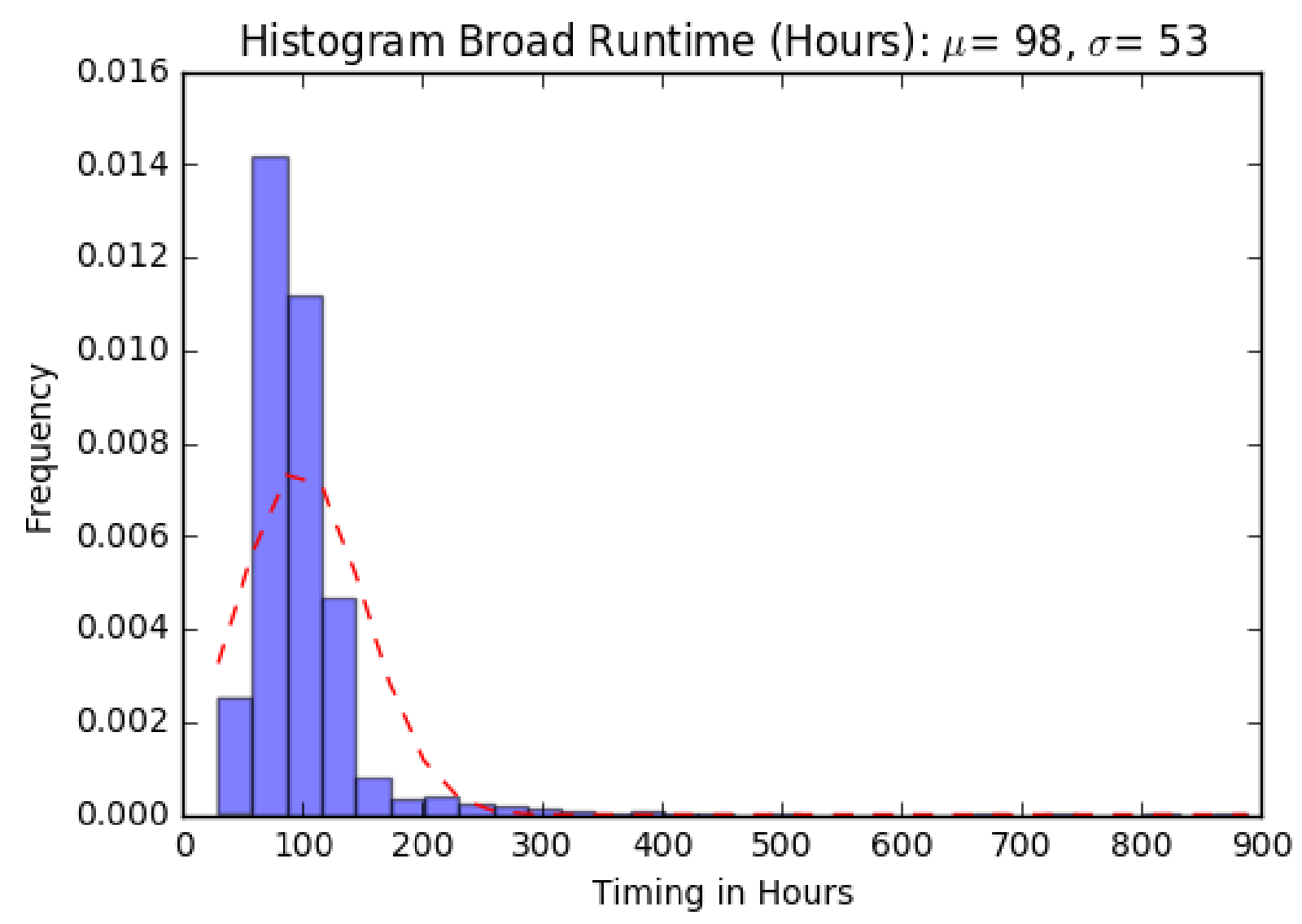

641

642 Supplementary Figure 10: Average runtime for the Broad somatic variant calling workflow.

643 Preceding the variant calling workflow, the GATK co-cleaning step takes an additional 24 hours.

644 
645 Supplementary Table 1. Percentage samples/donors run at each site for each pipeline 646

\begin{tabular}{|c|c|c|c|c|c|}
\hline & BWA & Sanger & DKFZ/EMBL & Broad/MuSE & OxoG \\
\hline AWS Ireland & 5.0 & 16.4 & 0.6 & & 31.1 \\
\hline Azure & 0.4 & 0.6 & 2.6 & 8.6 & \\
\hline BSC & 10.2 & 17.2 & 28.5 & & \\
\hline Collaboratory & & & & & 68.9 \\
\hline DKFZ (HPC) & & & 55.8 & & \\
\hline $\begin{array}{l}\text { DKFZ } \\
\text { (OpenStack) }\end{array}$ & 14.5 & 10.2 & 8.5 & & \\
\hline EMBL-EBI & 12.6 & 3.3 & & & \\
\hline ETRI & 2.1 & 5.8 & & & \\
\hline iDASH & & 4.8 & & & \\
\hline OICR & 1.8 & 5.6 & 1.0 & & \\
\hline PDC & 11.8 & 4.2 & & & \\
\hline Sanger & & 7.0 & 3.0 & & \\
\hline Seven Bridges & & & & 23.1 & \\
\hline UCSC & 30.6 & 13.0 & & 68.2 & \\
\hline UTokyo & 10.9 & 11.9 & & & \\
\hline
\end{tabular}


648 Supplementary Table 2. Data distribution as of May 2017. While ETRI GNOS and CGHub 649 served as data centres during the project, they have since been retired. Variant calls include 650 those from individual variant calling pipelines and the final consensus callsets. Long-term 651 repositories are denoted by asterisk $\left(^{*}\right)$ and will increase their data holdings over time while 652 GNOS servers are gradually being retired. Latest information can be found at 653 https://dcc.icgc.org/repositories

654

\begin{tabular}{|c|c|c|c|c|c|c|}
\hline \multirow[b]{2}{*}{ Data Repository } & \multicolumn{3}{|c|}{ ICGC Data } & \multicolumn{3}{|c|}{ TCGA Data } \\
\hline & $\begin{array}{l}\% \text { WG } \\
\text { Alignments } \\
\text { (534 TB) }\end{array}$ & $\begin{array}{l}\% \text { RNA-Seq } \\
\text { Alignments } \\
(13 \text { TB) }\end{array}$ & $\begin{array}{l}\% \text { Variant } \\
\text { calls } \\
(520 \text { GB) }\end{array}$ & $\begin{array}{l}\% \text { WG } \\
\text { Alignments } \\
\text { (240 TB) }\end{array}$ & $\begin{array}{l}\text { \% RNA-Seq } \\
\text { Alignments } \\
\text { (14 TB) }\end{array}$ & $\begin{array}{l}\% \\
\text { Variant } \\
\text { calls } \\
(228 \text { GB) }\end{array}$ \\
\hline BSC GNOS & 100.0 & 30.0 & 0.3 & & & \\
\hline DKFZ GNOS & 25.0 & & 62.9 & & & \\
\hline EMBL-EBI GNOS & 100.0 & 59.3 & 98.6 & & & \\
\hline UTokyo GNOS & 54.6 & 17.1 & 1.6 & & & \\
\hline $\begin{array}{l}\text { UChicago-ICGC } \\
\text { GNOS }\end{array}$ & 16.8 & 40.3 & 28.7 & & & \\
\hline $\begin{array}{l}\text { UChicago-TCGA } \\
\text { GNOS }\end{array}$ & & & & 100.0 & 100.0 & 100.0 \\
\hline$E G A^{*}$ & 97.8 & & & & & \\
\hline Collaboratory* & 100.0 & 100.0 & 100.0 & & & \\
\hline AWS* & 76.7 & 80.1 & 75.1 & & & \\
\hline Bionimbus PDC* & & & & 100.0 & 100.0 & 0.2 \\
\hline
\end{tabular}


656

The following set of tables show how costs are calculated for Figure 5 which compares the costs and accuracies of running the different combination of variant calling pipelines.

Supplementary Table 3a. The average run time for each workflow was rounded up to the nearest hour to reflect how AWS charges for EC2 instances that run for part of an hour. The size of the output files are noted as they contribute to either egress or storage costs.

\begin{tabular}{|c|c|c|c|}
\hline Workflow & $\begin{array}{c}\text { Average wall clock } \\
\text { run time (hours) }\end{array}$ & $\begin{array}{c}\text { Size of output files } \\
\text { (GB) }\end{array}$ & $\begin{array}{c}\text { AWS EC2 Instances } \\
\text { Used }\end{array}$ \\
\hline BWA-Mem & 140 & 134 & m1.xlarge \\
\hline Sanger & 53 & 2 & r3.8xlarge \\
\hline DKFZ/EMBL & 41 & 5 & r3.8xlarge \\
\hline Broad & 89 & 35 & r3.8xlarge \\
\hline OxoG & 4 & 1.5 & m2.4xlarge \\
\hline
\end{tabular}

Supplementary Table $3 \mathbf{b}$. The project utilized EC2 spot instances in US East (N. Virginia), US West (Oregon), EU (Ireland) regions. Because spot pricing fluctuates, users should consult real-time information. The average spot pricing listed here was based on our own usage throughout the project.

\begin{tabular}{|c|c|c|c|c|}
\hline $\begin{array}{c}\text { AWS EC2 } \\
\text { Instances }\end{array}$ & vCPU & Mem (GiB) & Storage (GB) & Average spot pricing \\
\hline m1.xlarge & 4 & 15 & $4 \times 420$ & $\$ 0.0426$ \\
\hline r3.8xlarge & 32 & 244 & $2 \times 320$ & $\$ 0.3382$ \\
\hline m2.4xlarge & 8 & 68.4 & $2 \times 840$ & $\$ 0.0834$ \\
\hline
\end{tabular}

Supplementary Table 3c. Cost calculations are based on the above spot pricing and an egress cost of $\$ 0.09$ per GB. The analysis time is made up of 3 steps: (1) running the BWA-Mem workflow on two separate instances to align simultaneously one tumor and one normal specimen; (2) running the variant calling workflows simultaneously with the longest running workflow dictating the run time of this step; (3) running the OxoG workflow after all variant calling workflows are completed. If analyzing 100 donors with all 3 variant calling pipelines, the analysis will involve running a fleet of 200,300 and $100 \mathrm{EC} 2$ instances, respectively in the 3 steps. We have no other significant storage cost as the reference files amount to $35 \mathrm{~GB}$ costing under $\$ 1 /$ month in S3. An alternative to transferring the data out is to store the $312 \mathrm{~GB}$ of data for each donor in S3 for under $\$ 8 /$ month. 


\begin{tabular}{|c|c|c|c|c|c|}
\hline $\begin{array}{l}\text { Variant Calling } \\
\text { Pipelines }\end{array}$ & $\begin{array}{l}\text { Total } \\
\text { Cost }\end{array}$ & $\begin{array}{l}\text { Compute } \\
\text { Cost }\end{array}$ & $\begin{array}{l}\text { Egress } \\
\text { Cost }\end{array}$ & $\begin{array}{l}\text { Analysis } \\
\text { Time } \\
\text { (days) }\end{array}$ & $\begin{array}{l}\text { Median Sensitivity, } \\
\text { Precision, F1 }\end{array}$ \\
\hline All 3 pipelines & 102.19 & 7.15 & 28.04 & 9.7 & $\begin{array}{l}0.9047+/-0.03145 \\
0.9348+/-0.03785 \\
0.9151+/-0.02820\end{array}$ \\
\hline Sanger only & 54.63 & 30.19 & 24.44 & 8.2 & $\begin{array}{l}0.8032+/-0.06515 \\
0.9550+/-0.03855 \\
0.8629+/-0.04795\end{array}$ \\
\hline $\begin{array}{l}\text { DKFZ/EMBL } \\
\text { only }\end{array}$ & 50.84 & 26.13 & 24.71 & 7.7 & $\begin{array}{l}0.7565+/-0.0544 \\
0.9352+/-0.0365 \\
0.8313+/-0.05125\end{array}$ \\
\hline Broad only & 69.77 & 42.36 & 27.41 & 9.7 & $\begin{array}{l}0.9095+/-0.01955 \\
0.8386+/-0.06335 \\
0.8687+/-0.04085\end{array}$ \\
\hline $\begin{array}{l}\text { Sanger \& } \\
\text { DKFZ/EMBL }\end{array}$ & 68.94 & 44.05 & 24.89 & 8.2 & $\begin{array}{l}\frac{\text { Union }}{0.8454}+/-0.0572 \\
0.9032+/-0.04405 \\
0.8669+/-0.0509 \\
\text { Intersect } \\
0.7228+/-0.05385 \\
0.9954+/-0.00980 \\
0.8216+/-0.04390\end{array}$ \\
\hline Sanger \& Broad & 87.88 & 60.29 & 27.59 & 9.7 & $\begin{array}{l}\frac{\text { Union }}{0.9374+/-0.01935} \\
0.8183+/-0.06395 \\
0.8653+/-0.04220 \\
\frac{\text { Intersect }}{0.7856+/-0.0566} \\
0.9913+/-0.0111 \\
0.8632+/-0.03755\end{array}$ \\
\hline $\begin{array}{l}\text { DKFZ/EMBL \& } \\
\text { Broad }\end{array}$ & 84.09 & 56.23 & 27.86 & 9.7 & $\begin{array}{l}\frac{\text { Union }}{0.9339+/-0.01955} \\
0.801+/-0.06505 \\
0.8576+/-0.0429 \\
\text { Intersect } \\
0.7384+/-0.05865 \\
0.9939+/-0.0186 \\
0.8315+/-0.0456\end{array}$ \\
\hline
\end{tabular}


682 Supplementary Table 4. DOIs for PCAWG core analysis workflows

683

684

\begin{tabular}{|c|c|c|c|c|}
\hline Workflow/Tool & Dockstore & Latest DOI & Version & Github \\
\hline $\begin{array}{l}\text { pcawg-bwa- } \\
\text { mem-workflow }\end{array}$ & $\begin{array}{l}\frac{\text { https://dockstore }}{\text { org/containers/q }} \\
\text { uay.io/pancance } \\
\text { r/pcawg-bwa- } \\
\text { mem-workflow }\end{array}$ & 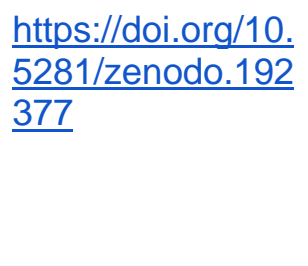 & 2.6.8_1.2 & $\begin{array}{l}\frac{\text { https://github.co }}{\text { m/ICGC-TCGA- }} \\
\text { PanCancer/Seq } \\
\frac{\text { ware-BWA- }}{\text { Workflow }}\end{array}$ \\
\hline $\begin{array}{l}\text { pcawg-dkfz- } \\
\text { workflow }\end{array}$ & $\begin{array}{l}\frac{\text { https://dockstore }}{\text { org/containers/q }} \\
\text { uay.io/pancance } \\
\text { r/pcawg-dkfz- } \\
\text { workflow }\end{array}$ & $\frac{\underline{\text { https://doi.org/10 }}}{\underline{\underline{5281 / \text { zenodo. } 19}}}$ & 2.0.1_cwl1.0 & $\begin{array}{l}\frac{\text { https://github.co }}{\text { m/lCGC-TCGA- }} \\
\frac{\text { PanCancer/DE }}{\text { WrapperWorkflo }} \\
\underline{w}\end{array}$ \\
\hline $\begin{array}{l}\text { pcawg-sanger- } \\
\text { cgp-workflow }\end{array}$ & $\begin{array}{l}\frac{\text { https://dockstore }}{\text {.org/containers/q }} \\
\frac{\text { uay.io/pancance }}{\text { r/pcawg-sanger- }} \\
\text { cgp-workflow }\end{array}$ & $\frac{\frac{\text { https://doi.org/10 }}{.5281 / \text { zenodo. } 19}}{\underline{\underline{2162}}}$ & 2.0 .3 & $\begin{array}{l}\frac{\text { https://github.co }}{\text { m/ICGC-TCGA- }} \\
\text { PanCancer/CGP } \\
\text {-Somatic-Docker }\end{array}$ \\
\hline $\begin{array}{l}\text { pcawg_delly_wo } \\
\text { rkflow }\end{array}$ & $\begin{array}{l}\frac{\text { https://dockstore }}{\text {.org/containers/q }} \\
\text { uay.io/pancance } \\
\text { r/pcawg delly w } \\
\text { orkflow }\end{array}$ & $\frac{\frac{\text { https://doi.org/10 }}{.5281 / \text { zenodo. } 19}}{\underline{\underline{2166}}}$ & 2.0.1-cwl1.0 & $\begin{array}{l}\frac{\text { https://github.co }}{\text { m/ICGC-TCGA- }} \\
\frac{\text { PanCancer/DE }}{\text { WrapperWorkflo }} \\
\underline{w}\end{array}$ \\
\hline \multicolumn{5}{|l|}{ broad } \\
\hline oxog & & & & \\
\hline
\end{tabular}

